\title{
Acute Lengthening of Progenitor Mitosis Influences Progeny Fate during Cortical Development in vivo
}

\author{
Aaron Mitchell-Dick ${ }^{\mathrm{a}}$ Andrea Chalem ${ }^{\mathrm{a}}$ Louis-Jan Pilaz ${ }^{\mathrm{a}-\mathrm{c}}$ Debra L. Silver ${ }^{\mathrm{a}}$ d-f \\ ${ }^{a}$ Department of Molecular Genetics and Microbiology, Duke University Medical Center, Durham, NC, USA;

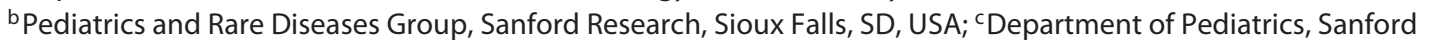 \\ School of Medicine, University of South Dakota, Sioux Falls, SD, USA; d Department of Cell Biology, Duke University \\ Medical Center, Durham, NC, USA; ${ }^{\mathrm{e} D e p a r t m e n t}$ of Neurobiology, Duke University Medical Center, Durham, NC,

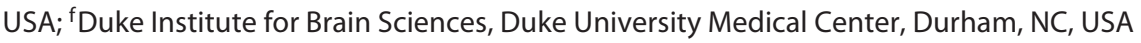

\section{Keywords}

Mitosis $\cdot$ Progenitor $\cdot$ Cortex $\cdot$ Cell fate $\cdot$ Neurogenesis

\begin{abstract}
Background/Aims: Prenatal microcephaly is posited to arise from aberrant mitosis of neural progenitors, which disrupts both neuronal production and survival. Although microcephaly has both a genetic and environmental etiology, the mechanisms by which dysregulation of mitosis causes microcephaly are poorly understood. We previously discovered that prolonged mitosis of mouse neural progenitors, either ex vivo or in vitro, directly alters progeny cell fate, resulting in precocious differentiation and apoptosis. This raises questions as to whether prolonged progenitor mitosis affects cell fate and neurogenesis in vivo, and what are the underlying mechanisms? Methods/Results: Towards addressing these knowledge gaps, we developed an in vivo model of mitotic delay. This uses pharmacological inhibition to acutely and reversibly prolong mitosis during cortical development, and fluorescent dyes to label direct progeny. Using this model, we discovered that a causal relationship between mitotic delay of neural progenitors and altered prog-
\end{abstract}

\section{KARGER}

(c) 2020 S. Karger AG, Basel

karger@karger.com

www.karger.com/dne eny cell fate is evident in vivo. Using transcriptome analyses to investigate the state of delayed cells and their progeny, we uncovered potential molecular mechanisms by which prolonged mitosis induces altered cell fates, including DNA damage and $\mathrm{p} 53$ signaling. We then extended our studies to human neural progenitors, demonstrating that lengthened mitosis duration also directly alters neuronal cell fate. Conclusions: This study establishes a valuable new experimental paradigm towards understanding mechanisms whereby lengthened mitosis duration may explain some cases of microcephaly.

(c) 2020 S. Karger AG, Basel

\section{Introduction}

The cerebral cortex is a highly ordered and complex structure which is critical for our higher cognitive functions including analytical thought and complex learning and language. In the developing mouse cortex, neurons are generated between embryonic day (E)10.5 and E18.5 [1]. In mice, cortical excitatory neurons are primarily generated from radial glial cells (RGCs). RGCs can pro-

Debra L. Silver

Department of Molecular Genetics and Microbiology

Duke University Medical Center, 224 CARL Building, Box 3175 DUMC

Durham, NC 27710 (USA)

debra.silver@duke.edu 
duce neurons directly, or indirectly via production of intermediate progenitors (IPs), which themselves divide one to two times before generating neurons [2-4]. In mice, RGCs can undergo symmetric proliferative divisions generating two new RGCs, symmetric neurogenic divisions generating two new neurons, or asymmetric divisions generating any of the following pairs: RGC/IP; RGC/neuron; neuron/IP. RGCs produce cortical excitatory neuron subtypes sequentially with the earliest-born neurons populating deep cortical layers VI-V and the late-born neurons residing in the upper layers IV-II. Neurogenesis is influenced by many parameters, including cell cycle kinetics of progenitors [5, 6]. For example, over the course of development mitosis (M phase) increases [7] and shortened S phase is linked to differentiation [8]. Further, manipulation of either G1- or M-phase kinetics impacts neurogenesis [9-11].

Genetic or environmental perturbations to embryonic cortical development can result in devastating neurodevelopmental disorders, including microcephaly and autism spectrum disorder [12-14]. Individuals diagnosed with microcephaly have reduced head circumference more than 3 standard deviations below the mean. Microcephaly is accompanied by varying degrees of cognitive deficiency. Primary microcephaly is present at birth in contrast to postnatal degenerative microcephaly which may have a distinct etiology. While autosomal recessive primary microcephaly is relatively rare, microcephaly syndromes are highly prevalent, affecting between 1 and $2 \%$ of the population. To date, autosomal recessive primary microcephaly has been linked to mutations in 25 loci [15]. Remarkably, the vast majority of these genes encode proteins associated with cell division [15]. This has led to a long-standing hypothesis in the field that disruptions to mitosis explain microcephaly. Indeed, a link between aberrant mitosis and microcephaly has been borne out in human organoid, cell culture, and mouse models. In human organoid models, defective progenitor mitosis is associated with altered neuron and progenitor number and massive apoptosis [16, 17]. Further, mouse studies of human microcephaly genes reveal consistent phenotypes of excessive apoptosis as well as imbalanced numbers of more neurons and fewer progenitors [18-24].

Beyond a genetic basis, microcephaly is also caused by viral infection, notably Cytomegalovirus or Zika virus (ZIKV) infection during pregnancy [25]. Several studies have shown that acute ZIKV infection can also impair neural progenitor mitosis and in some cases is associated with aberrant neurogenesis and apoptosis in human and mouse models [26-29]. Thus, both genetic and viral

Prolonged Progenitor Mitosis in Cortical Development causes of microcephaly are linked to progenitor mitosis defects and similar alterations in neurogenesis and cell survival. However, despite this clear clinical link, the mechanisms by which mitotic defects underlie this pathology are poorly understood.

We previously uncovered one potential explanation for how progenitor mitotic defects could cause microcephaly, with the discovery that progenitors undergoing a delayed mitosis (specifically M phase) directly produce aberrant cell progeny $[11,30]$. Using live imaging of brain slices and primary progenitors from $\mathrm{Magoh}^{+/-}$microcephalic brains, we determined that mitotically delayed mutant progenitors produced significantly fewer neural progenitors and more apoptotic progeny. We extended this correlative data by employing two distinct drugs, STLC and nocodazole, to reversibly and acutely prolong mitosis in wild-type (WT) ex vivo organotypic brain slices and in vitro primary progenitors.In both paradigms, progenitors with prolonged mitosis alone directly generated neurons at the expense of progenitors; exceedingly long mitoses led to the production of apoptotic progeny. While these data indicate prolonged mitosis is relevant for microcephaly, they raise several important questions. Is a causal link between prolonged mitosis and altered neurogenesis evident in vivo? How do alterations in mitotic length drive rapid changes in differentiation? Do these mechanisms hold true in human progenitors?

To address these questions, in this study we developed and characterized a new in vivo model for prolonged progenitor mitosis during cortical development. With this experimental paradigm, an acute injection of a pharmacological mitotic inhibitor into the embryonic cortex delayed mitosis of progenitors transiently, reversibly, and reproducibly. This was coupled with FlashTag, to selectively label the delayed progenitors and their direct progeny. Using this strategy to lineage trace daughter cells of delayed progenitors, we discovered that acute mitotic delay of RGCs in vivo results in reduced progenitor number, concomitant increased neuron number, as well as apoptosis of progeny. We then isolated the delayed progenitors and progeny to assess early transcriptome changes associated with a single mitotic delay in the context of otherwise normal development. Among the observed transcriptome changes, our data highlight p53 signaling as a notable pathway linked to mitotic delay, with wellstudied p53 targets prominently upregulated. Finally, using human embryonic stem cell (ESC)-derived neural progenitors, we demonstrate that mitotic delay is also linked to altered cell fates in human progenitors similar to that observed in vitro and in vivo in the mouse. Taken 
together, our study further establishes prolonged mitosis as a driver of apoptosis and neuronal fates in vivo and reinforces that increased mitotic length provides a plausible mechanism to explain some cases of microcephaly. Our study thus describes and characterizes a valuable new model for understanding the etiology of microcephaly.

\section{Materials and Methods}

\section{Mice}

All mice were fed a standard chow. All alleles were maintained on a C57BL/6J background. Emx $1^{\text {Cre }}, B a x^{-/-}$and $p 53^{\text {lox/lox }}$ mice were acquired from Jackson labs. Plug dates were defined as E0.5 on the morning of observed plugging. All animal use was approved by Duke IACUC.

\section{Mouse Injections}

Intraventricular injections were performed on E14.5 embryos. Pregnant dams were anesthetized with Isoflurane. $0.8 \mu \mathrm{L}$ of DMSO or STLC $(80,150,250 \mu \mathrm{M}$, dissolved in DMSO) with $0.01 \%$ Fast Green in $\mathrm{dH}_{2} \mathrm{O}$ was injected into the right ventricle of each embryo using a kd Scientific Legato 100 microinjector. For some experiments (Fig. 1, 2a-h, 3, 5, 6; online suppl. S1; for all online suppl. material, see www.karger.com/doi/10.1159/000507113) we co-injected with $0.8 \mu \mathrm{L}$ of $1 \mathrm{~mm}$ FlashTag (CFSE CellTrace ${ }^{\circledR}$, Thermo Fisher C34570, stock prepared according to the manufacturer's recommendations then diluted $1 / 10$ in injection solution). For some experiments (Fig. 2i-k, 4; online suppl. S2-S4) we performed a sequential injection in which STLC or DMSO was first injected followed $2 \mathrm{~h}$ later by FlashTag injection into the same brain. Briefly, between surgeries, the dam was placed in her home cage and allowed to recover. For the second injection, sutures were removed, injection was performed, then the surgical opening was again sutured closed. The dam was placed in her home cage and allowed to recover. Both surgeries were performed in the same manner. The sequential injection was optimal to label cells at the time of their delay in prometaphase at the ventricular surface. At various time points post-injection embryos were dissected, and cortices were dissected, isolated, and prepared for downstream applications described below.

\section{Fluorescence-Activated Cell Sorting of FlashTag+ Cells and Cell Cycle Analysis}

Embryos were dissected into ice-cold HBSS (Life Technologies, Without $\mathrm{Ca}$ or $\mathrm{Mg}$ ). Cortices were isolated and meninges removed in ice-cold HBSS. As necessary, tails were reserved from embryos for genotyping and placed in lysis buffer. Cortices were placed one or two hemispheres at a time into $250 \mu \mathrm{L}$ of cold $0.25 \%$ trypsin/ EDTA (Life Technologies) with RNase-Free DNase I (New England Biolabs, 1:1,000). Cortices were incubated in trypsin for 10 $\min$ at $37^{\circ} \mathrm{C}$. Trypsin was inactivated with addition of $500 \mu \mathrm{L}$ of ice-cold HBSS containing propidium iodide (PI), and tissue was passed through a glass pipette (VWR) 6 times, then passed through a $30 \mu \mathrm{m}$ filter. Dissociated cells were sorted at $6{ }^{\circ} \mathrm{C}$ with gates for forward scatter (FSC), side scatter (SSC), PI, and CFSE (carboxyflourescin succinimidyl ester), with dead cells and doublets discarded, and live cells collected in either HBSS (without Ca or Mg) with $10 \%$ FBS, HBSS (no FBS), RLT buffer (Qiagen), or Tri-Reagent (Sigma). For cell cycle analysis, cells were sorted at $4^{\circ} \mathrm{C}$ into ice-cold HBSS with $10 \%$ FBS. Cells were spun at $200 \mathrm{~g}$ at $4^{\circ} \mathrm{C}$, supernatant removed, and resuspended in $300 \mu \mathrm{L} \mathrm{PBS}$ (no Ca or Mg); $650 \mu \mathrm{L}$ of $100 \% \mathrm{EtOH}$ was added dropwise to fix cells. Cells were fixed for $1 \mathrm{~h}$, then spun down at $200 \mathrm{~g}$ and washed $3 \times$ with $5 \%$ BSA (fraction V; Thermo Fisher) in $1 \times$ PBS (no Ca or Mg). Cells were incubated with RNAse A 1:1,000 (Sigma) for $30 \mathrm{~min}$ at room temperature, and PI added at 1:1,000. Cells were incubated for a further $30 \mathrm{~min}$ at room temperature then stored at $4{ }^{\circ} \mathrm{C}$ until sorted. Cells were sorted and analyzed using a FACSCantoII ${ }^{\mathrm{TM}}$ Flow Cytometer (BD Biosciences) capturing FSC, SSC, CFSE, and PI. Output files were analyzed in FlowJo Version X. Cell cycle graphs were created in FlowJo. Briefly, output files were gated for single cells comparing FSC versus SSC and PI intensity plotted in a histogram to determine the proportion of $2 \mathrm{~N}, \mathrm{~S} / \mathrm{G} 2$, and $4 \mathrm{~N}$ cells. For $+2 \mathrm{~h}$, we isolated the top 1.5-2.3\% of CFSE+ cells for control and the top $14-20 \%$ of CFSE + cells for STLC. For $+9 \mathrm{~h}$, we isolated the top 25\% of CFSE+ cells for control and for STLC.

\section{qPCR of FACS-Sorted Cells}

Cells were sorted into RLT buffer and lysates frozen at $-80^{\circ} \mathrm{C}$. Samples were thawed for $15 \mathrm{~min}$, mixed, and RNA was purified using Qiagen RNEasy Mini Plus columns into $15 \mu \mathrm{L}$ elution buffer (Qiagen). cDNA was generated using iScript (Bio-Rad). qPCR was performed using template-specific primers with iScript SYBR GREEN (Bio-Rad) in 15- $\mu \mathrm{L}$ reactions in an Applied Biosystems StepOnePlus or in 2- $\mu \mathrm{L}$ reactions performed in Applied Biosystems QuantStudio6 Flex. For $2-\mu \mathrm{L}$ reactions, a qPCR plate was loaded from a source plate using a manual template on a LabCyte Echo 550 Acoustic Liquid Handler. Primers used:

$\beta$-actin: AGATCAAGATCATTGCTCCT, CCTGCTTGCTGATCCACATC; Ccng1 (Cyclin-G1): TTGGCTTTGACACGGAGACA, AAGCAGCTCAGTCCAACACA; Cdkn1a (p21): TGACAGATTTCTATCACTCCAAGC, ACTTTAAGTTTGGA GACTGGGAGA; Tcf19: GAACTCCAAGGGGACGACTG, GG CCAAAGGTCAGAAGGTCA; Neurog1: ATC ACC ACT CTC TGA CCC, GAG GAA GAA AGT ATT GAT GTT GCC TTA.

\section{Immunofluorescence}

Embryonic brains were fixed overnight in $4 \% \mathrm{PFA}$ at $4^{\circ} \mathrm{C}$, then suspended in $30 \%$ sucrose at $4{ }^{\circ} \mathrm{C}$ for at least $4 \mathrm{~h}$. Brains were submerged in Neg- $50^{\mathrm{TM}}$ (Thermo Scientific) and frozen on dry ice, then placed at $-80{ }^{\circ} \mathrm{C} ; 20-\mu \mathrm{m}$ sections were cryosectioned and slides were stored at $-80^{\circ} \mathrm{C}$. Sections were washed with PBS and incubated in $0.25 \%$ Triton for $15 \mathrm{~min}$. If antigen retrieval was performed, sections were then submerged in sodium citrate buffer, heated to $100^{\circ} \mathrm{C}$ for $30 \mathrm{~min}$, taken off heat, and allowed to equilibrate to room temperature for $\sim 30 \mathrm{~min}$. Then sections were blocked in $1 \times$ PBS with $10 \%$ NGS for $1 \mathrm{~h}$. Primary antibodies (listed in online suppl. S4 Table S3), were incubated at room temperature for $2 \mathrm{~h}$. Sections were washed $3 \times$ and then secondary antibodies, along with DAPI, were incubated for $20 \mathrm{~min}$. Sections were washed and mounted in Mowiol. Immunofluorescence images were acquired by epifluorescence on a Zeiss AxioObserver with Apotome or on a Zeiss 780 inverted confocal microscope, and images were analyzed in Fiji software. Fiji quantitative data were transferred into Excel spreadsheets and statistical analyses were performed with GraphPad Prism. 


\section{Quantifications}

In 150-, 200-, or 250- $\mu \mathrm{m}$ cortical columns of the dorsal cortex the number of cells expressing FlashTag (FT+ cells) and markers for RGCs (Pax6/Sox2), IPs (Tbr2), or neurons (NeuroD2) were calculated using a Fiji Cell Counter. For layer marker analysis, in $250-\mu \mathrm{m}$ cortical columns the abundance and colocalization of FT and various pyramidal neuron markers were quantified. For prometaphase and mitosis quantifications, phosphohistone $\mathrm{H} 3$ (PH3)-positive cells with condensed DNA were counted in blinded sections, and the number of mitotic cells ( $\mathrm{PH} 3+$ cells with condensed DNA) and prometaphase cells ( $\mathrm{PH} 3+$, DAPI without a metaphase plate, not in anaphase or telophase) was determined as follows: (number of cells $/ \mu \mathrm{m}) \times 100$. For $\gamma \mathrm{H} 2 \mathrm{AX}$ analysis, immunofluorescence was performed using antigen retrieval, and images were captured on a Zeiss 780 inverted confocal microscope. Zsections were acquired every $2 \mu \mathrm{m}$. All processing and analysis were performed in ImageJ (Fiji); 2 Z-sections of each image were combined in a maximum intensity projection for analysis. The phase of mitosis was named for each cell using DAPI. For analysis of metaphase and prometaphase cells, an oval of $25-$ to $26-\mu \mathrm{m}$ perimeter was drawn around each cell. For analysis of anaphase cells an ellipse of 28- to 29- $\mu \mathrm{m}$ perimeter was drawn around each cell. Mean, minimum, and maximum intensity of pixels were measured. For $\gamma \mathrm{H} 2 \mathrm{AX}$, analysis was completed on sections prepared 3 $\mathrm{h}$ after injection, and for pATR, analysis was completed $4 \mathrm{~h}$ after injection. For the STLC condition, all FT+ cells at the ventricle were measured. For DMSO, FT+ and FT-cells were measured.

\section{EdU Time Course}

E14.5 embryos were injected as described (mouse injections), first with DMSO or STLC, and $2 \mathrm{~h}$ later with FlashTag; $6 \mathrm{~h}$ after injection, EdU was administered by IP injection at $10 \mathrm{mg} / \mathrm{kg}$ every $2 \mathrm{~h}$ until $18 \mathrm{~h}$ to cumulatively label dividing cells in this period; 24 $\mathrm{h}$ later, cortices were dissected, fixed in 4\% PFA, and sectioned (as above). Prior to antibody staining, slides containing sections were washed twice in $1 \times$ PBS for 8 min each, permeabilized with $0.5 \%$ Triton in $1 \times$ PBS for $2 \mathrm{~min}$, washed 3 times in $1 \times$ PBS for $2 \mathrm{~min}$, and immunofluorescence staining for EdU was carried out using Life Technologies Click-It Plus Kit (\#C10749) following the manufacturer's instructions.

\section{Transcriptome Analysis}

E14.5 embryos were injected with FlashTag and DMSO or STLC using the sequential labeling approach described above. Following treatment and labeling of RGCs, at +2 and +9 h following delay induction cortices were dissected and RGCs dissociated as described above. FT+ cells were sorted into RLT lysis buffer and stored at $-80^{\circ} \mathrm{C}$. RNA was purified from sorted RGCs using the RNeasy Plus Micro Kit (Qiagen) and stored at $-80^{\circ} \mathrm{C}$. cDNA libraries were prepared using Nugen Universal Plus mRNA-seq and sequenced using an Illumina Hiseq 4000. RNA-seq data were processed as previously described [30]. In short, we used the TrimGalore toolkit which employs Cutadapt to trim low-quality bases and Illumina sequencing adapters from the $3^{\prime}$ end of the reads. Only reads that were $20 \mathrm{nt}$ or longer after trimming were kept for further analysis. Reads were mapped to the GRCm38v73 version of the mouse genome and transcriptome using the STAR RNA-seq alignment tool. Reads were kept for subsequent analysis if they mapped to a single genomic location. Gene counts were compiled using the HTSeq tool. Only genes that had at least 10 reads in any given li- brary were used in subsequent analysis. Normalization and differential expression were carried out using the DESeq2 Bioconductor package with the R statistical programming environment. Within individual analysis, genes were further filtered if more than 4 samples had zero reads. The false discovery rate was calculated to control for multiple hypothesis testing. Standard gene set enrichment analysis was performed using normalized counts for the entire gene set averaged from biological triplicates per condition to identify gene ontology terms and pathways associated with altered gene expression for each of the comparisons performed. Gene identification was matched to Ensembl mouse CHIP file IDs.

\section{Primary Cultures}

Live imaging of RGCs and quantifying cell fate was performed as previously described, with slight modifications [11,30]. Briefly, before dissection, primary culture medium was assembled and MatTek glass coverslip bottom culture plates were coated with 50 $\mathrm{ug} / \mathrm{mL}$ poly-L-lysine for at least $30 \mathrm{~min}$ at $37^{\circ} \mathrm{C}$. Plates were washed $3 \times$ with sterile water. The primary culture medium recipe $-500 \mu \mathrm{L}$ N2 supplement (Thermo Fisher), 1 mL B27 supplement (without vitamin A; Thermo Fisher), $500 \mu \mathrm{L}$ of $100 \mathrm{mM}$ N-acetyl-cysteine (prepared in sterile water), and $50 \mu \mathrm{L}$ of $10 \mathrm{ug} / \mathrm{mL}$ mouse bFGF (R\&D Systems 3139-FB025, prepared using the manufacturer's instructions) - was added to $48.95 \mathrm{~mL}$ of cold high-glucose DMEM (with sodium pyruvate, glutamine; Thermo Fisher 11995-065). The medium was kept at $4^{\circ} \mathrm{C}$ for up to 1 week. Only enough medium needed for each day was heated at $37^{\circ} \mathrm{C}$. E13.5 cortices were dissected in ice-cold HBSS, and the meninges removed, before placing the cortices into ice-cold $0.25 \%$ trypsin-EDTA (Gibco). Cortices were digested for $10 \mathrm{~min}$ at $37^{\circ} \mathrm{C}$, then trypsin was inactivated by adding $1 \times$ trypsin inhibitor (Glycine max soybean; Sigma) diluted in primary cell culture medium. Cells were spun down at $200 \mathrm{~g}$ for $5 \mathrm{~min}$, and supernatant removed. Cells were washed once with primary culture medium, supernatant was removed, and cells were diluted in primary cell culture medium. Cells were counted with a cell counter, and 250,000 cells were plated onto MatTek plates with glass coverslip bottoms in $1 \mathrm{~mL}$ of primary culture medium. The culture plate was placed in a $37^{\circ} \mathrm{C}$ incubator with $5 \% \mathrm{CO}_{2}$, and cells were allowed to settle and attach to the plate bottom for $1 \mathrm{~h}$ before proceeding.

\section{In vitro Live Imaging}

A Zeiss Axiovision inverted A1 microscope fitted with a Pecon incubation chamber was warmed to $37^{\circ} \mathrm{C}$ and atmosphere conditioned to $5 \% \mathrm{CO}_{2}$ via sensors and integrated Zen software. For drug application, each drug was diluted to the appropriate concentration in $500 \mu \mathrm{L}$ of warmed primary culture medium (STLC $1 \mu \mathrm{M}$, AZ20 $1 \mu \mathrm{M}, \mathrm{VE} 821160 \mathrm{nM}$ ); $500 \mu \mathrm{L}$ of primary culture media was removed from cultures, and medium with DMSO, STLC, individual ATR inhibitors, or drug cocktail was added to each well, respectively. The culture plate was placed into the microscope under a Pecon plate cover attached to a regulated, humidified $\mathrm{CO}_{2}$ line providing $5 \% \mathrm{CO}_{2}$. Typically, 80-120 $x, y$, $z$ imaging positions were chosen in Zen software ( 20 per well), and DIC images acquired with 10 -min intervals between frames. The $z$-focus was periodically adjusted as needed. Cells were incubated for $3 \mathrm{~h}$, then STLC (STLC wash off) was removed from all conditions by removal of medium, $1 \times$ wash with primary culture medium, followed by application of new medium. New medium containing either DMSO or ATR inhibitors was added, and the plate was taken back to the 


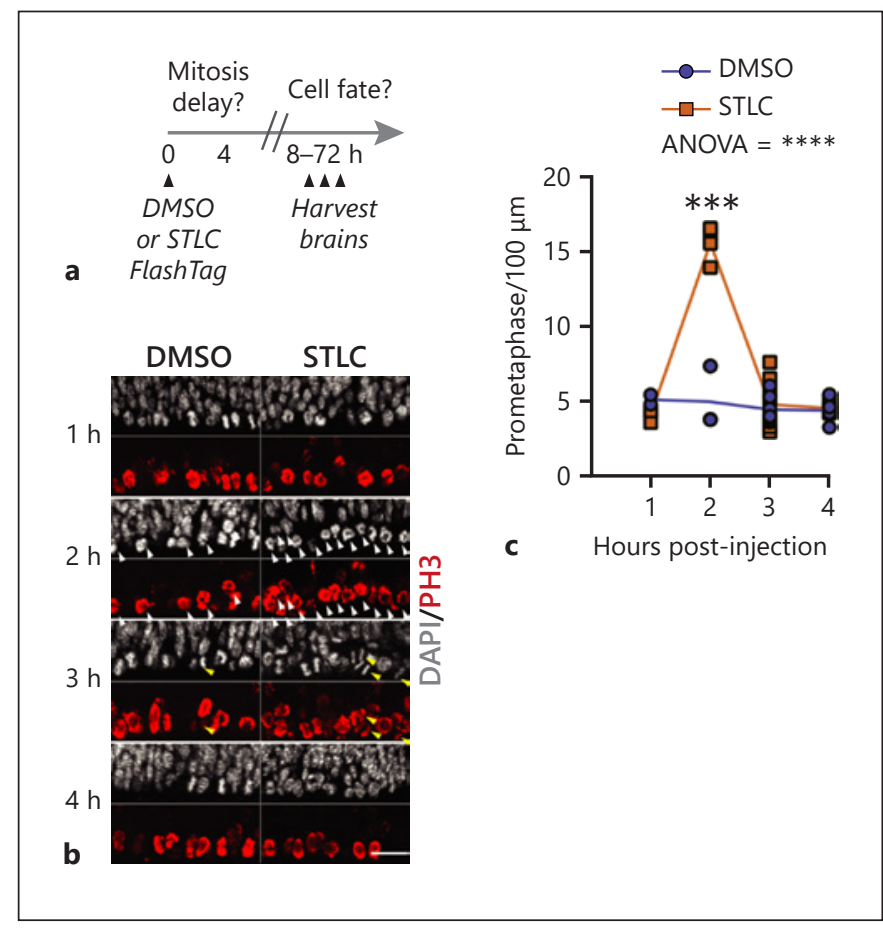

Fig. 1. A model for acute inducible prolonged mitosis in vivo. a Experimental paradigm with injection of either DMSO or STLC at time point 0 and harvesting of brains for fate analysis between 8 and $72 \mathrm{~h}$ later. b Immunofluorescence depicting PH3+ cells (red) and DAPI (white) at the ventricle of an E14.5 cortex, at different time points following injection of DMSO or STLC. Scale bar, 25 $\mu \mathrm{m}$. White arrowheads: prometaphase cells; yellow arrowheads: anaphase cells. c Quantification of the average number of prometaphase cells per $100 \mu \mathrm{m}$, along the ventricular surface of the cortex. $n \geq 3$ embryos for each condition, at each timepoint. $n=$ 37 total embryos. Statistics: ANOVA, with a post hoc Student's $t$ test. ${ }^{* * *} p=0.0005 .{ }^{* * * *} p<0.0001$. Error bar $=\mathrm{SD}$.

microscope to resume imaging. After an additional $3 \mathrm{~h}$, ATR inhibitors were removed (ATRi wash off) by replacing all the cell culture medium. The culture plate was taken back to the microscope to resume imaging, $z$-focus was adjusted after $30 \mathrm{~min}$, and imaging proceeded for $16 \mathrm{~h}$.

\section{In vitro Fixation and Fate Analysis}

Following live imaging capture, acquisition was paused (to maintain $x, y, z$ positions), the culture plate was removed, medium was removed, and cells were fixed in $4 \%$ PFA for $20 \mathrm{~min}$ at $4{ }^{\circ} \mathrm{C}$. Cells were washed $3 \times$ in $1 \times$ PBS for 2 min each. Cells were permeabilized in $0.1 \%$ Triton for 10 min. Cells were washed $2 \times$ in $1 \times$ PBS for 2 min each, then blocked in 3\% BSA in $1 \times$ PBS for $30 \mathrm{~min}$. Cells were then incubated with primary antibodies diluted in $3 \%$ BSA $/ 1 \times$ PBS for $1-2 \mathrm{~h}$ at room temperature. Cells were washed $3 \times$ with $1 \times$ PBS for 5 min each. Cells were incubated in secondary antibody with DAPI for 20 min at room temperature, then washed $3 \times$ in $1 \times$ PBS for 5 min each, finally adding $500 \mu \mathrm{L}$ PBS to each well prior to image acquisition. The plate was then placed back into the incubation chamber on the microscope, allowed to equilibrate to chamber temperature for $30 \mathrm{~min}$, and the $z$-plane was adjusted for each $x, y$ position. Images were acquired in Zen software. Fixed imaging was then correlated to the last frame of live imaging to call fates for individual daughter cell pairs. The total number of frames in which a cell was in mitosis was multiplied by 10 to achieve mitosis duration. This was correlated to cell fates of daughters and the data compiled in Microsoft Excel. Data were analyzed in Excel and graphed in Excel or GraphPad Prism.

\section{Differentiation and Live Imaging of Human Neural Progenitor} Cells

Human neural progenitor cells (hNPCs) were differentiated from ESC or iPSCs as previously described [31]. Usage was approved as exempt by Duke IRB. Briefly, ESCs or iPSCs were cultured until 100\% confluent on Matrigel-coated 6-well plates in mTesR medium. Cells were differentiated by replacing mTesR medium with hNPC differentiation medium (1:1 N2/B27 medium with dual SMAD inhibitors Dorsomorphin and SB431542); $2.5 \mathrm{~mL}$ of medium was replaced daily for 10 days. Cells were then passaged with Dispase and plated onto $60-\mathrm{mm}$ dishes coated with polyornithine (Sigma) and laminin (Sigma) in $1 \mathrm{~mL}$ of N2/B27 with Dorsomorphin, SB431542, and ROCK inhibitor (Y-27632; StemCell Technologies. The next day, the medium was replaced with N2/ B27 medium. Cells were maintained in this medium until formation of neural rosettes was observed, then the medium was supplemented with bFGF for up to 4 days. bFGF was removed around day 18-20, and cells were cultured for an additional 2 days. Cells were passaged with accutase and plated in N2/B27 medium onto $60-\mathrm{mm}$ plates coated with polyornithine and laminin. Cells were passaged with accutase at 1:4 ratio, maintaining cultures at around $50-80 \%$ confluence. For live imaging, cultures between differentiation days 24 and 36 were plated onto polyornithine/laminincoated MatTek 24-well glass-bottom plates.

\section{Results}

\section{A Paradigm for in vivo Delay of Progenitor Mitosis}

Increased mitotic length of embryonic neural progenitors is associated with microcephaly, yet the underlying mechanisms by which mitosis duration impairs neurogenesis are poorly understood. Further, whether prolonged progenitor mitosis alters cell fate in vivo is unknown. To address this gap, we developed an in vivo experimental paradigm to acutely lengthen mitosis duration of progenitors in the developing mouse cortex (Fig. 1a).

To acutely prolong progenitor mitosis, we injected the small molecule STLC (S-trityl-L-cysteine) directly into the ventricle of the developing cortex at E14.5. STLC specifically and potently inhibits activity of the kinesin Eg5, which is critical for bipolar spindle formation [32]. Importantly, STLC application reversibly arrests cells in prometaphase and has been widely used to induce prometaphase delay in immortalized cell lines without affecting other cell cycle stages. Furthermore, our previous 
studies showed that acute STLC treatment of embryonic brain slices or primary progenitor cultures was sufficient to prolong mitosis without inducing nonspecific cell death or aneuploidy [11].

In an asynchronous dividing population, we predicted that injection of STLC would result in a buildup of mitotic cells at the ventricle in a dosage-dependent fashion. To test this, we first optimized STLC drug dosage to induce a short mitotic delay in vivo. For this, we injected either DMSO or STLC into the ventricle of E14.5 embryos using volumes of $0.8 \mu \mathrm{L}$ at a starting concentration of $80 \mu \mathrm{M}$ (Fig. 1). We then assessed the kinetics of mitosis by quantifying mitotic cells at $1,2,3$, and $4 \mathrm{~h}$ following injection of $0.8 \mu \mathrm{L}$ of $80 \mu \mathrm{M}$ STLC (Fig. 1b). To quantify prometaphase ventricular cells, we stained sections for $\mathrm{PH} 3$ expression and DAPI. We specifically measured progenitors dividing at the ventricle (presumed RGCs) which were $\mathrm{PH} 3+$ and showed condensed DNA, characteristic of prometaphase [11] (Fig. 1b). Cells were quantified over a 100- $\mu$ m-wide radial column (Fig. 1c). In STLC-treated brains, the number of prometaphase RGCs at the ventricle significantly increased $2 \mathrm{~h}$ after injection, then largely receded by 3 and $4 \mathrm{~h}$, when the fraction of ventricular progenitors in mitosis had returned to control levels. In addition to measuring prometaphase we extended our analysis to all mitotic stages by quantifying cells for $\mathrm{PH} 3$ expression and the presence of condensed DNA (online suppl. Fig. S1C). This revealed a peak of mitosis at $2 \mathrm{~h}$ which was sustained at $3 \mathrm{~h}$, relative to control. Importantly, at $3 \mathrm{~h}$, this likely demarcates $\mathrm{PH} 3+$ cells which are transiting anaphase (Fig. 1a, yellow arrowheads). Thus, the number of ventricular RGCs in mitosis increased at 2 and $3 \mathrm{~h}$ after injection and resolved to normal rates at $4 \mathrm{~h}$.

To reinforce whether this was an appropriate concentration at which to use STLC, we tested higher dosages and measured mitosis at $4 \mathrm{~h}$. Notably, the number of prometaphase cells was significantly higher with increasingly higher STLC concentration, indicating a correlated dosage response to the Eg5 inhibitor in vivo (online suppl. Fig. S1A, B). Mitotically delayed cells were evident both apically at the ventricle and basally in the higher concentrations. While these higher concentrations provide a more potent delay of mitosis, they may be less physiologically relevant than the delay induced with $80 \mu \mathrm{M}$. Thus, for remaining studies we opted to use $80 \mu \mathrm{M}$. These data establish a reproducible paradigm to acutely and reversibly extend the length of mitosis, and specifically prometaphase, in RGCs of the in vivo developing mouse cortex.

Prolonged Progenitor Mitosis in Cortical Development
Acute Mitotic Delay of RGCs Alters Progenitor and Neuronal Fate of Progeny in vivo

Having established an assay to acutely and reversibly delay RGCs in mitosis, we next aimed to examine the effects of this acute cell cycle alteration. To label mitotic RGCs and their progeny we employed FlashTag, also termed CFSE. Previous studies have demonstrated the utility of FlashTag in the developing cortex for labeling RGCs adjacent to the ventricle, and in their progeny $[33,34]$. Importantly, because RGCs at the ventricle are largely in G2/M, this allows us to label mitotic RGCs. Indeed, we were able to recapitulate utility of this labeling paradigm (Fig. 2a).

With this dual mitotic delay and labeling paradigm, we then sought to assess the direct effects of prolonged mitosis on RGCs in vivo (Fig. 2b). Our prior ex vivo and in vitro studies indicate that prolonged progenitor mitosis directly alters cell fate [11]. Thus, we hypothesized that mitotic delay in vivo would alter the neuronal and progenitor fates of progeny in the cortex. In the E14.5 cortex, RGCs cycle about $19 \mathrm{~h}$ on average, and divide either symmetrically or asymmetrically to produce RGCs, IPs, and excitatory neurons $[8,35]$. We unilaterally injected STLC or DMSO (control) along with FlashTag into the cortices of E14.5 embryos. We then quantified the fate of FT+ daughter cells $16 \mathrm{~h}$ after injection, reasoning this would allow us to examine the immediate progeny of delayed and labeled progenitors. Colocalization of FT with markers for RGCs (Pax6) and IPs (Tbr2) was quantified. At 16 $\mathrm{h}$ post-injection, in control FT+ progeny, about $50 \%$ of cells were Pax6+ (Fig. 2c, d). Compared to DMSO, in STLC treated brains, there was a significant $24.6 \%$ decrease in FT+Pax6+ RGCs $(p=0.01)$. In contrast, there was no significant change in newborn Tbr2+ IP number between control and STLC conditions $(p=0.24)$ (Fig. 2e, $f)$. These data indicate that mitotic delay in vivo preferentially reduces the number of RGCs, without having a striking impact upon IP number.

Given the significant reduction of RGC number following a prolonged progenitor mitosis we next asked whether there was a concomitant increase in newborn daughter neurons. To monitor newborn neurons, we quantified the expression of NeuroD2, a neurogenic transcription factor [36]. NeuroD2 is expressed in the VZ, SVZ, and cortical plate soon after neurons are born. In control brains about $60 \%$ of FT+ newborn progeny were NeuroD2 positive (Fig. 2g, h). The proportion of daughter cells expressing NeuroD2 showed a slight, but significant 1.14-fold increase following prolonged prometaphase $(p<0.0001)$. This increase was especially evident in rostral regions of the developing cortex (online suppl. Fig.

Dev Neurosci 2019;41:300-317 305 


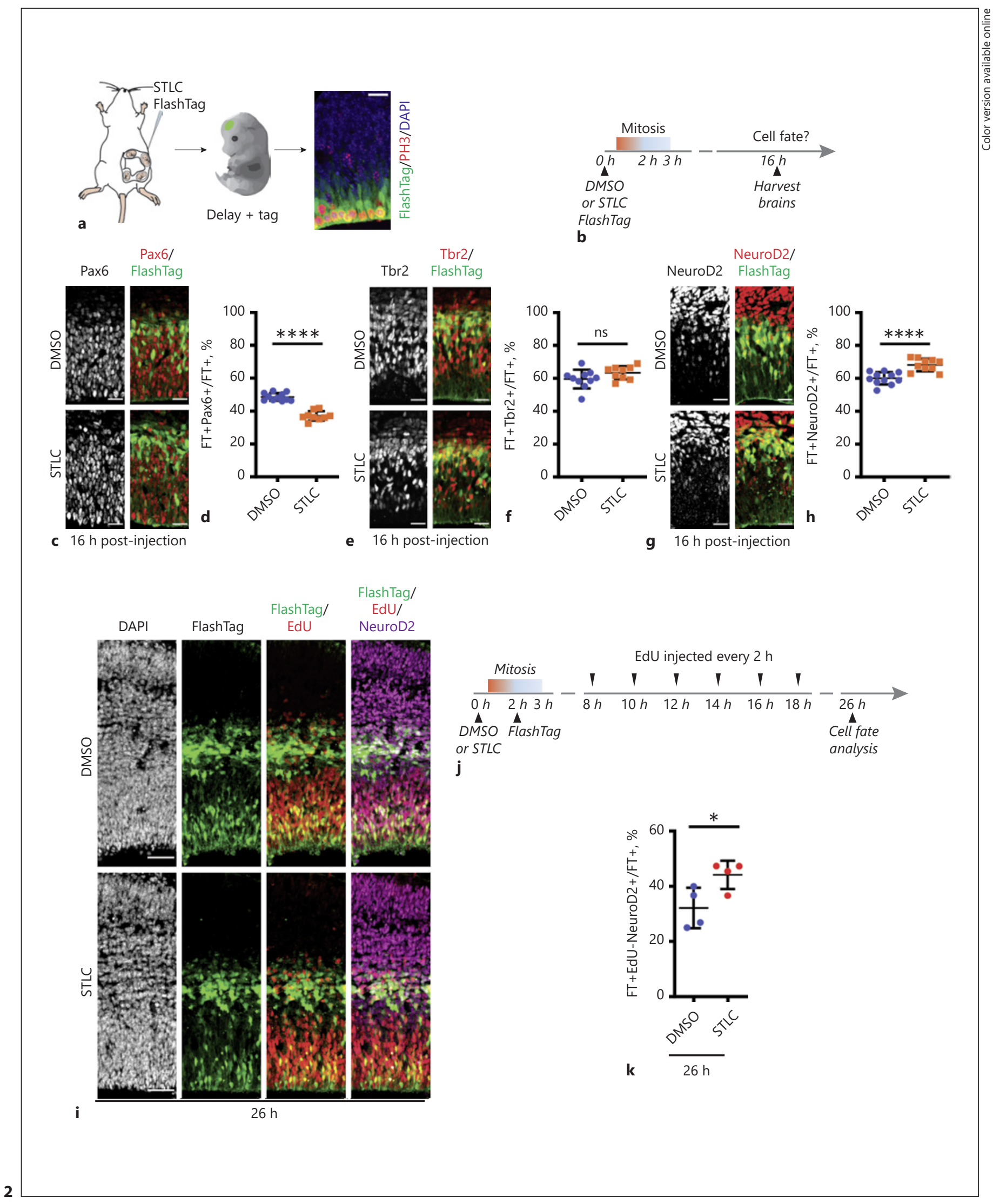

(For legend see next page.) 
S1D). Low expression of NeuroD2 can also label Tbr2+ IPs. However, we observed no change in Tbr2+ number following prolonged prometaphase, and thus posit that an increased number of NeuroD2+ cells reflects more newborn neurons.

We aimed to further probe this possibility and discriminate between NeuroD2+ proliferative versus differentiated daughter cells. To assess the number of newborn neurons following prolonged mitosis, we labeled proliferative daughters using cumulative EdU injection every 2 $\mathrm{h}$, starting $8 \mathrm{~h}$ after injection of either STLC or DMSO and FlashTag (Fig. 2i, j). With this approach, any FT+ daughters exiting the cell cycle would be EdU-, while proliferative progeny would be EdU+. We quantified the proportion of NeuroD2+EdU-FT+ cells that exited the cell cycle $26 \mathrm{~h}$ post-injection (Fig. 2k). Compared to control, in STLC-treated brains, there was a significant increase in cell cycle exit of NeuroD2+ cells (compare $32 \%$ to $44 \%$, respectively, $p=0.035$ ). Thus, prolonged mitosis of RGCs alters neurogenesis in vivo, resulting in fewer progenitors and more neurons. Notably, these phenotypes of skewed proliferative to neurogenic fates are commonly seen in microcephaly disease models [18-24].

Given the pro-neurogenic outcome linked to increased mitosis duration, we next asked whether acute mitotic delay would result in a longer-term impact upon cortical fates, by quantifying laminar markers $72 \mathrm{~h}$ after injection. In order to discriminate direct and indirect progeny derived from delayed progenitors, we performed cumulative EdU labeling following either STLC or DMSO injection [37] (online suppl. Fig. S2A). We quantified SATB2 and $\operatorname{ROR} \beta$ as these primarily mark neurons of layers II/ III and IV, respectively, which are generated at or after E14.5, when mitotic delay was induced [38]. Although we noted slight trends towards more upper-layer neurons, these were not quite significant (online suppl. Fig. S2B, C). This suggests that an acute lengthening of mitosis in RGCs in this paradigm is not sufficient to dramatically alter lamination patterns of cortical neurons.

Fig. 2. Effects of prolonged mitosis in vivo on cell fate acquisition. a Scheme of injection paradigm used in this study. $\mathbf{b}$ Time course of injection paradigm used to assess cell fate following prolonged mitosis. c, e, g Images depicting E14.5 brains $16 \mathrm{~h}$ after injection with either STLC or DMSO and stained for Pax6 (white, red) (c), Tbr2 (white, red) (e), Neurod2 (white, red) (g) along with FlashTag (FT, green). d, f, h Quantification of the fraction of FT+ cells which are Pax6 (d), Tbr2 (f), and Neurod2 (g) $16 \mathrm{~h}$ after injection of DMSO or STLC. Pax6 analysis, DMSO: $n=11$ embryos; STLC: $n=9$ embryos, $n=2$ sections each. Tbr2 analysis, DMSO: $n=10$
Acute Mitotic Delay of RGCs Independently Increases Apoptosis of Newborn Progeny in vivo

Another phenotype commonly linked to microcephaly is apoptosis. Hence, we asked whether cell death of daughter cells was evident following prolonged mitosis of RGCs. Analysis of brains $8 \mathrm{~h}$ after STLC injection revealed apoptotic-like puncta evident in FT+ cells, but not in controls (Fig. 3a). To monitor apoptosis, we performed immunostaining for cleaved caspase 3 (CC3) at 8 and $16 \mathrm{~h}$ after injection of DMSO or STLC (Fig. 3a). $8 \mathrm{~h}$ postinjection, CC3 expression was significantly increased in $22 \%$ of FT+ daughter cells in STLC compared to control $(p=0.0021)$ (Fig. 3b). CC3 expression was highly specific to FT+ cells $(92.2 \pm 2.7 \%)$ (Fig. $3 \mathrm{c}$ ). This indicates that STLC injection does not cause a non-cell autonomous increase in apoptosis in the tissue, similar to that seen in our prior studies [11]. By $16 \mathrm{~h}$ after prolonged mitosis, $\mathrm{CC} 3+\mathrm{FT}+$ cells were only evident in some STLC brains, and thus not significantly different than control. However, in STLC-treated brains, the average number of apoptotic progeny was significantly different between 8 and $16 \mathrm{~h}$. These data indicate that apoptosis of newborn progeny is high $8 \mathrm{~h}$ after injection. Based upon the kinetics of mitotic delay (Fig. 1, online suppl. S1), this indicates that apoptosis is evident in progeny which are estimated to be on average about $5 \mathrm{~h}$ old.

One possible explanation for reduced progenitor number and increased neuron number $16 \mathrm{~h}$ after injection is selective cell death of progenitors. To assess this, we quantified the fraction of CC3 cells which expressed a neuronal marker. Given that apoptotic CC3+ cells lose nuclear structure we used a cytoplasmic neuronal marker, Tuj $1 ; 16 \mathrm{~h}$ after injection, in the small number of apoptotic cells present, we quantified similar fractions of Tuj1positive and Tuj1-negative FT+ progeny (Fig. 3d, e). This suggests that apoptosis affects progenitors and neurons equivalently at a time point when cell fate is altered. We could not quantify the fates of cells that may have died earlier given the variable onset of fate marker expression.

embryos, STLC: $n=9$ embryos, $n=2$ or more sections each. NeuroD2 analysis, DMSO: $n=11$ embryos, STLC: $n=10$ embryos, $n=2$ or more sections each. Statistics: Student's $t$ test. i Images depicting FT (green), EdU (red), and NeuroD2 (purple) 26 h postinjection of DMSO or STLC. $\mathbf{j}$ EdU labeling scheme following injection of DMSO or STLC; analysis at $26 \mathrm{~h}$ post-injection. k Quantification of the fraction of FT cells which are EdU-NeuroD2+FT+ $26 \mathrm{~h}$ after injection of DMSO or STLC. NeuroD2: ${ }^{* *} p=0.0037$. Pax6: ${ }^{* * * *} p<0.0001$. EdU/NeuroD2: ${ }^{*} p=0.04$. Scale bars, a, 20 $\mu \mathrm{m} ; \mathbf{c}, \mathbf{e}, \mathbf{g}, 25 \mu \mathrm{m} ; \mathbf{i}, 100 \mu \mathrm{m}$. Error bar $=\mathrm{SD}$. 


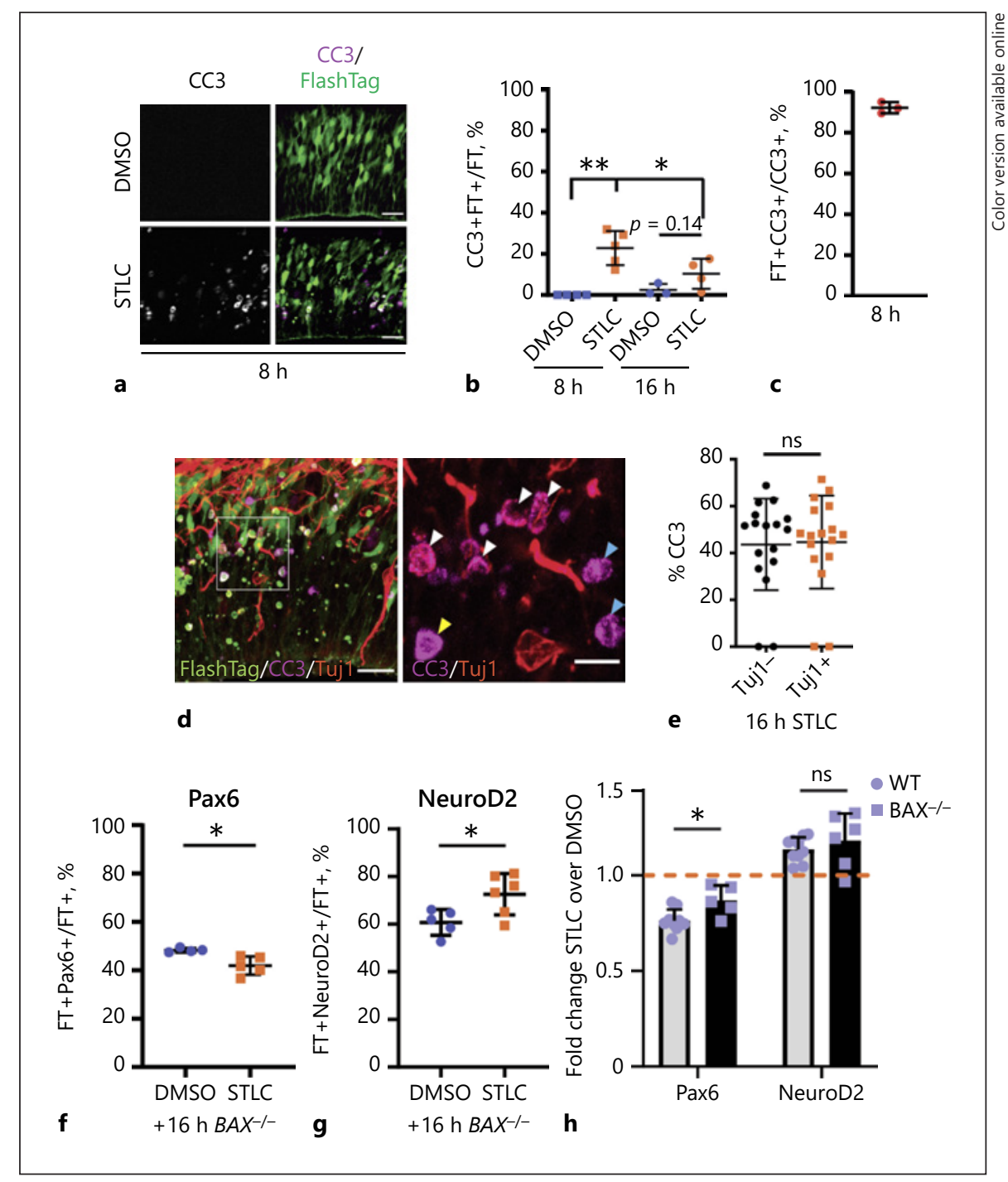

Fig. 3. Prolonged mitosis in vivo results in apoptosis in daughter cells. a Images depicting the VZ/SVZ with FT (green) and CC3 (white) $8 \mathrm{~h}$ after injection of DMSO or STLC. $\mathbf{b}$ Quantification of the fraction of FT cells which are CC3+FT+ cells in 200- $\mu$ m-wide radial columns. c Quantification of $\mathrm{CC} 3+$ cells that are $\mathrm{FT}+$ at $8 \mathrm{~h}$ in STLC condition. Dots represent individual embryos, $n=2$ or more sections each. d Images depicting the VZ/SVZ $+16 \mathrm{~h}$ after injection of STLC, depicting FT (green), CC3 (purple), and Tuj1 (red). Inset ROI depicting CC3 and Tuj1. White arrowheads: FT+CC3+Tuj1+; yellow arrowheads: FT+CC3+Tuj1-; blue arrowheads: FT+CC3+Tuj1-. e Quantification of the fraction of $\mathrm{CC} 3+$ cells that are either Tuj1+ or Tuj1- $16 \mathrm{~h}$ after STLC injec-

Nevertheless, these results are in line with the finding that prolonged mitosis directly promotes neuron generation, reduces progenitor production, and causes excess apoptosis in progeny. tion, in $200-\mu \mathrm{m}$-wide radial columns. $n>10$ sections. $\mathbf{f}$, $\mathbf{g}$ Quantification of the fraction of FT+ cells which are Pax6 (f) or NeuroD2 (g) $16 \mathrm{~h}$ after injection of DMSO or STLC in E14.5 Bax-/- embryos, in 200- $\mu \mathrm{m}$-wide radial columns. Pax6 analysis, DMSO: $n=$ 4 embryos; STLC: $n=5$ embryos, $n=3$ sections each. NeuroD2 analysis, DMSO: $n=5$ embryos, STLC: $n=6$ embryos, $n=3$ sections each. $\mathbf{h}$ Comparison of fold changes in expression of Pax6 or NeuroD2 between WT and Bax-/- embryos at $16 \mathrm{~h}$ following STLC injection. CC $38 \mathrm{~h}:{ }^{* * *} p=0.001,8 \mathrm{~h}$ vs. $16 \mathrm{~h}: p=0.05$. BAX Pax6: ${ }^{*} p=0.015$, NeuroD2: ${ }^{*} p=0.03$. Pax6 BAX vs. WT: ${ }^{*} p=$ 0.01 . Student's $t$ test. Scale bars, a, d, $25 \mu \mathrm{m} ; \mathbf{d}, 10 \mu \mathrm{m}$. Error bar $=$ $\mathrm{SD}$.

How does apoptosis affect the overall composition of progeny following prolonged progenitor mitosis? We further probed the distinct fates of apoptosis and differentiation by measuring the impact of prolonged mitosis in a $\mathrm{Bax}^{-/-}$model. These models are valuable for elimi- 
nating apoptosis, especially that which occurs during normal development [39]. Thus, we used this model to ask whether it could eliminate apoptosis associated with prolonged mitosis. We repeated either DMSO or STLC injections and collected brains for immunofluorescence analysis $16 \mathrm{~h}$ later. There was a significantly reduced fraction of Pax6+FT+ cells in Bax ${ }^{-/-}$animals compared to DMSO-treated $\mathrm{Bax}^{-/-}$mice (Fig. 3f). Concomitantly, there was an increased proportion of NeuroD2+ daughter cells from progenitors stalled in prometaphase (Fig. 3g). While the relative fold changes in NeuroD2+ cells were similar in WT and $\mathrm{Bax}^{-/-}$, the Pax6+ reduction following STLC was less striking in the Bax mutant compared to control (Fig. 3h). We again noted variable apoptosis in the $\mathrm{Bax}^{-/-}$brains $16 \mathrm{~h}$ after STLC treatment, similar to that seen in WT brains. Thus, it is possible that Bax does not completely eliminate apoptosis, perhaps due to redundancy with other apoptotic regulators. Nonetheless, the quantification of equivalent apoptosis in progenitors and neurons suggests that the increase in neurons is not entirely explained by selective progenitor apoptosis.

\section{Prolonged Prometaphase Alters the Transcriptome of}

Mitotic Progenitors and Their Progeny

Having established that RGC mitotic delay alters the differentiation state and survival of progeny, we sought to understand the cascade of molecular events which accompany cell fate changes. Specifically, we asked what are the downstream alterations in the transcriptome following an acute prolonged progenitor prometaphase? We thus took advantage of our model system to assess gene expression changes as a result of a single delay event. To this end, we injected E14.5 brains with STLC or DMSO, followed by injection of FlashTag $2 \mathrm{~h}$ later (Fig. 4a, online suppl. S4, see Materials and Methods). This paradigm was used in order to label populations of RGCs at their peak of prometaphase delay (Fig. 1) and label their direct progeny (Fig. 4b). Importantly, this paradigm recapitulated the results observed previously with co-injection of FlashTag and STLC (compare Fig. 2 to online suppl. S4B). Further, this sequential injection paradigm resulted in higher-fold cell fate alterations (online suppl. Fig. S4B).

We isolated cortices either immediately after FlashTag injection to enrich for mitotic RGCs $(+2 \mathrm{~h})$ or several hours later to enrich for newborn progeny $(+9 \mathrm{~h})$. We then performed FACS, collecting 20,000 cells/condition or 40,000 cells/condition for +2 and +9 h, respectively (Fig. 4b, c). Cells were sorted based upon FT signal to en-

Prolonged Progenitor Mitosis in Cortical Development rich relatively equivalent cell populations using a 5\% threshold for control and 1\% for STLC (online suppl. Fig. S3A, B). Using PI staining we assessed DNA content as a proxy for cell cycle stage (Fig. 4b, c). Of those cells derived from either STLC or DMSO brains at $+2 \mathrm{~h}$, about $50 \%$ had a 4 N DNA content (indicative of $\mathrm{G} 2 / \mathrm{M}$ ) and about $50 \%$ had a 2N DNA content (indicative of G0/G1/S). Given the timing of mitotic delay, an important caveat of this approach is that the control would be expected to have later G1 stages relative to G1 in STLC. We then carried out transcriptome analysis in triplicate at each time point. With this we obtained $>5 \times 10^{6}$ reads/sample. We noted that between time points there was a significant increase in neurogenic transcripts in WT brains, correlating with increased differentiation of newborn daughter cells. This reflects the utility of our assay in labeling progenitors and newborn progeny (online suppl. Fig. S3C, and suppl. S2 Table S1).

At $+2 \mathrm{~h}$, we detected differential expression of a small number of transcripts including 221 transcripts $(\mathrm{q}<0.1)$, or 167 transcripts with a stricter statistical cutoff $(\mathrm{q}<$ 0.05) (Fig. 4e, f; online suppl. S2 Table S1). Of these, the majority of transcripts were downregulated (e.g., 100 in q $<0.05$ ). Gene set enrichment analysis (cutoff of FDR $<0.1$, fold change \pm 2 ) revealed several differentially expressed pathways (online suppl. S3 Table S2). This included several categories related to cell cycle including DNA replication, G2/M transition and G1. We also noted enrichment for DNA damage pathways, including several genes assigned to "S phase enrichment."

At $+9 \mathrm{~h}$, we detected less differential expression which included changes in 63 transcripts $(\mathrm{q}<0.1)$ or 41 transcripts $(\mathrm{q}<0.05)$ (Fig. $4 \mathrm{e}, \mathrm{g})$. Similar to $+2 \mathrm{~h}$, the majority of transcripts were upregulated (e.g., 29 in $\mathrm{q}<0.05$ ) (Fig. 4g). Gene set enrichment analysis (cutoff of FDR < 0.1 , fold change \pm 2 ) revealed several differentially expressed pathways including those linked to cell cycle and translation (online suppl. S3 Table S2). Strikingly, at +9 $\mathrm{h}$, the p53 pathway was significantly enriched in the upregulated transcript list. This overlaps with multiple studies which have discovered upregulation of p53 targets, including irradiation-induced microcephaly of the embryonic brain $[40,41]$.

To validate these findings qPCR was performed on transcripts from the +2 and +9 h time points. For the +2 $\mathrm{h}$ time point we assessed levels of Neurogl and Tcf19, two transcripts linked to differentiation and showing $>2$-fold change from RNA-seq. Both were significantly upregulated in STLC-treated brains relative to control, consistent with the transcriptomic findings (Fig. 4h). For the 

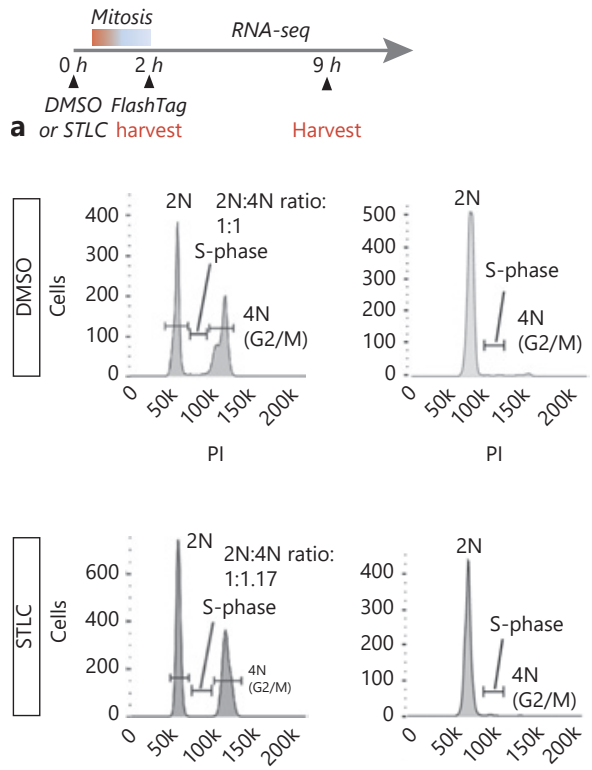

b

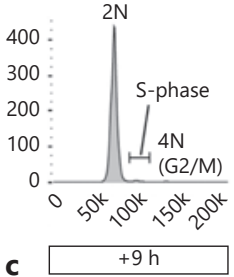

$2 \mathrm{~h}$

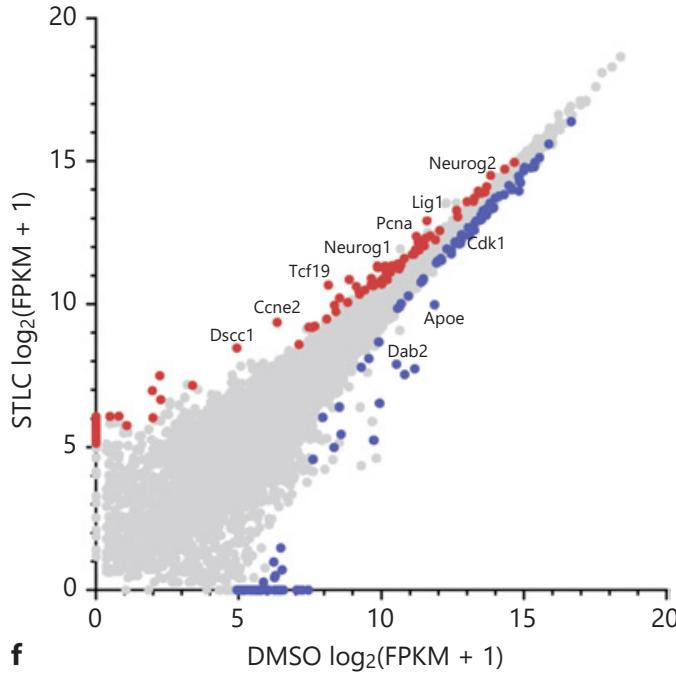

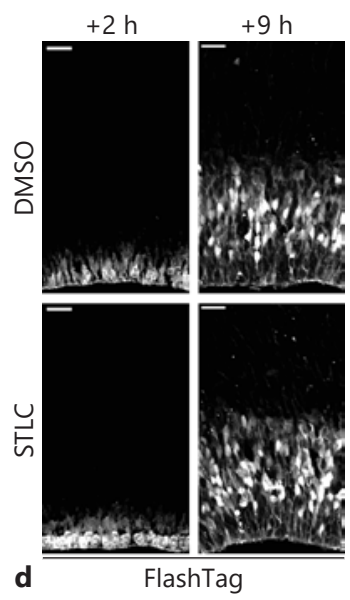
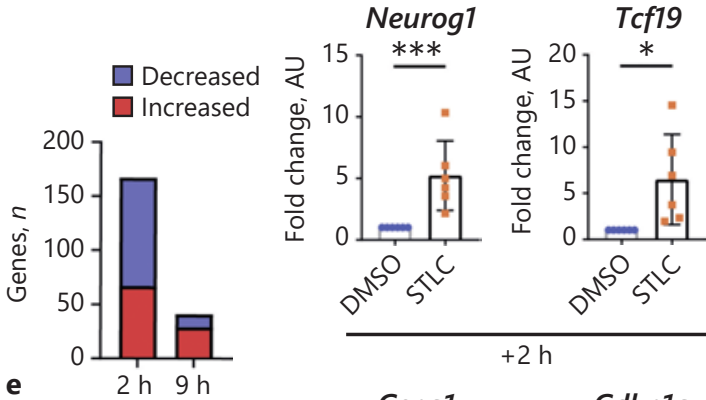

Cong1

Cdkn1a

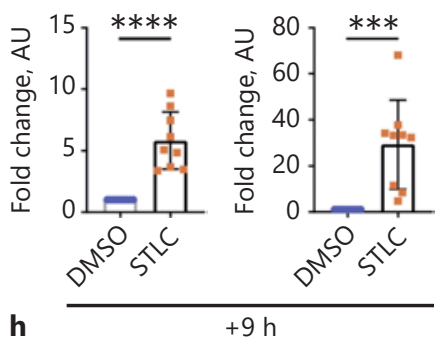

$9 \mathrm{~h}$

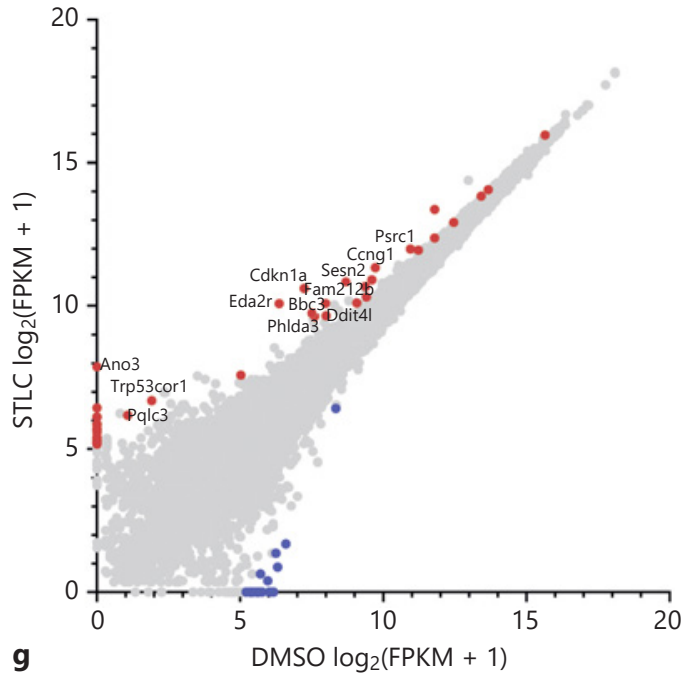

Fig. 4. Transcriptome analysis following prolonged mitosis. a Schematic of RNA-seq analysis. b, $\mathbf{c}$ Cell cycle plots of FACSsorted populations by condition and time point. $y$ axis: cell count, $x$ axis: propidium iodide. d Images depicting cortices stained for FT (white) 2 and $9 \mathrm{~h}$ after injection with either DMSO or STLC conditions, corresponding to RNA-seq time points. e Graph of total significant transcript changes at both timepoints, $>2$-fold expression difference STLC vs. DMSO, $p<0.05$. f, $\mathbf{g}$ Scatterplots of gene expression changes at time points $+2 \mathrm{~h}$ (f) and $+9 \mathrm{~h}(\mathbf{g})$. Red: $p<0.05$, enriched in STLC; blue: $p<0.05$, enriched in DMSO. Transcripts of notable change or associated with significant pathways have been annotated. $\mathbf{h}$ qPCR for select RNA-seq candidates at time points +2 and +9 h. Neurog1: ${ }^{* * *} p=0.004$, Tcf19: ${ }^{* * *} p=0.02$, Ccng1: ${ }^{* * *} p<0.0001$, Cdkn1a: $* * * p=0.0005$. Dots equal independent biological replicates. At $+2 \mathrm{~h}$, samples were identical to those used for FACs, whereas at $+9 \mathrm{~h}, 3$ brains were from FACS and the remaining were independent replicates. Error bar $=\mathrm{SD}$. 

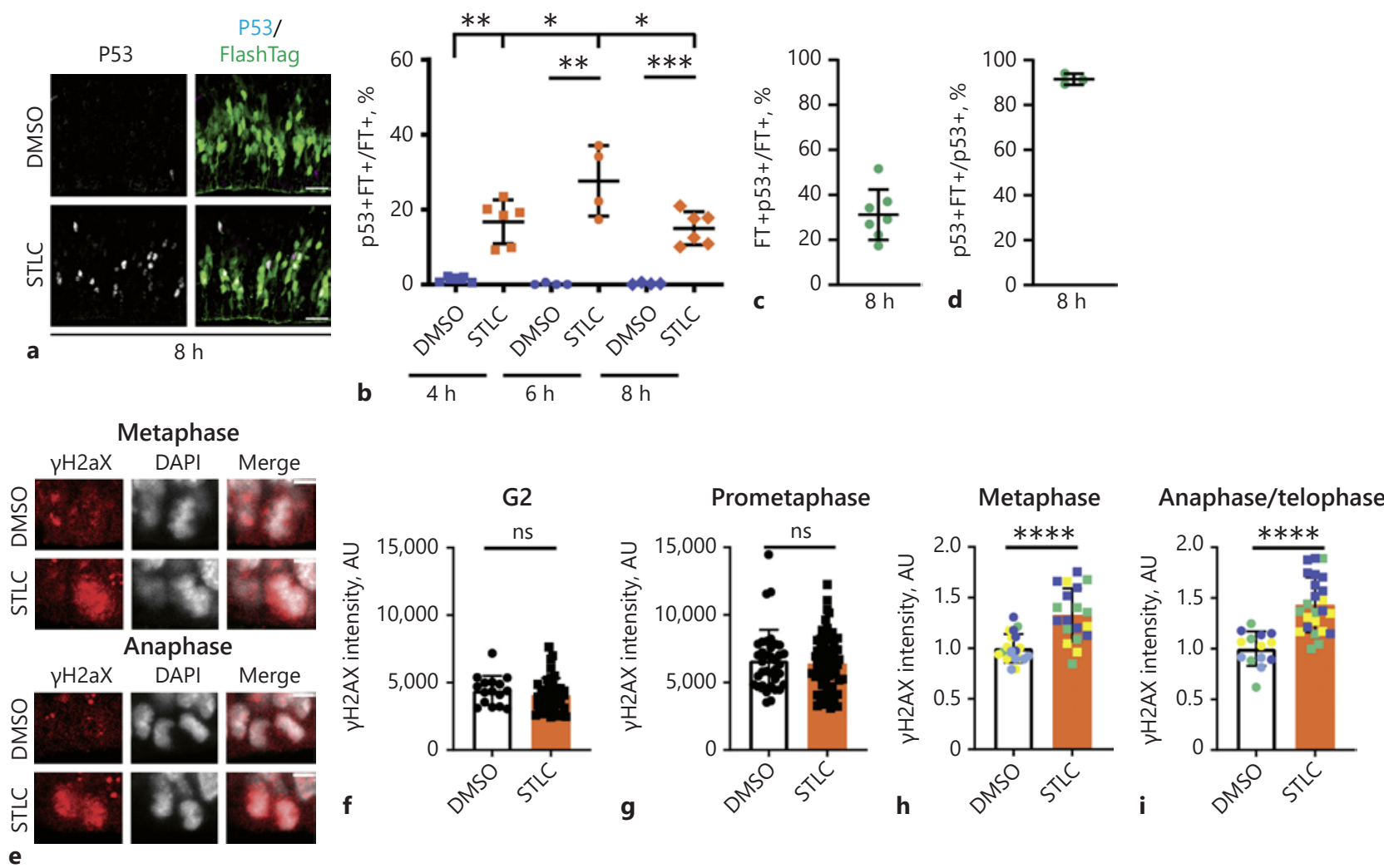

Fig. 5. Upregulated p53 pathway and DNA damage following prolonged mitosis. a Images of the VZ/SVZ depicting FT (green) and p53 (white) $8 \mathrm{~h}$ after injection of DMSO or STLC. $\mathbf{b}$ Quantification of the fraction of FT+ cells that are CC3+FT+ cells 4,6 , and $8 \mathrm{~h}$ after STLC injection via analysis of $200-\mu \mathrm{m}$ cortical columns. Dots represent individual embryos, average of 2 or more sections per embryo. 6 h: ${ }^{* *} p=0.004,8 \mathrm{~h}:{ }^{* * * *} p<0.0001,10 \mathrm{~h}:{ }^{* * *} p=0.0003$, $6 \mathrm{~h}$ vs. 8 h: ${ }^{*} p=0.02,8$ h vs. $10 \mathrm{~h}: p=0.04$. c Quantification of the fraction of FT + cells that are p53+ at $8 \mathrm{~h}$ in STLC condition. d Percent of p53+ cells labeled by FlashTag $8 \mathrm{~h}$ after STLC injection.

+9 h time point, we measured levels of $C d k n 1 a(P 21)$ and Cong1, which are known p53 targets. Both transcripts showed potent increases in STLC versus control, also consistent with results from RNA-seq at this time point (Fig. 4h).

In sum, transcriptome analysis of STLC-delayed RGCs and their progeny point to differential gene expression changes in just a small fraction of transcripts expressed in the E14.5 cortex, indicating that overall gene expression is largely unaffected. This provides a valuable and manageable list of transcripts which may be relevant for cell fate specification in response to mitotic delay.

Prolonged Progenitor Mitosis in Cortical Development $n=3$ embryos, average of 3 sections per embryo. e Images of $\mathrm{H} 2 \mathrm{AX}$ (red) and DAPI (white) in mitotic cells at the ventricle $3 \mathrm{~h}$ after injection of DMSO or STLC. $\mathbf{f}-\mathbf{i}$ Quantification of H2AX intensity of mitotic cells at the ventricle $3 \mathrm{~h}$ after DMSO or STLC injection. Dots represent individual cells across two or more embryos. Note that for STLC, primarily FT+ cells were quantified in order to enrich for mitotic cells. Statistics: Student's $t$ test. Metaphase: ${ }^{* * * *} p<$ 0.0001 , anaphase/telophase: ${ }^{* * *} p<0.0001$. Scale bars, a, $25 \mu \mathrm{m}$; e, $5 \mu \mathrm{m}$. Error bar $=$ SD.

\section{p53 Is a Major Signaling Pathway Altered following RGC Mitotic Delay in vivo}

The p53 pathway was strongly upregulated in newborn progeny following mitotic delay. We thus analyzed expression of stable nuclear $\mathrm{p} 53$ in brains following either DMSO or STLC injection, at $+4,+6$, and +8 h following injection of either DMSO or STLC (Fig. 5a, b). At all time points, p53 levels were significantly elevated in FT+ progeny, showing peak elevation $6 \mathrm{~h}$ after injection (Fig. 5b). p53 expression $8 \mathrm{~h}$ after injection was evident in $38 \%$ of FT+ daughter cells, when daughters are $\sim 5$ h old (Fig. $5 \mathrm{c}$ ). Importantly, p53 expression was also highly specific to FT + cells 8 h after injection $(91.5 \pm 2.4 \%, n=3)$ (Fig. $5 \mathrm{~d}$ ). We thus asked if $p 53$ loss would impact cell fate changes

Dev Neurosci 2019;41:300-317 


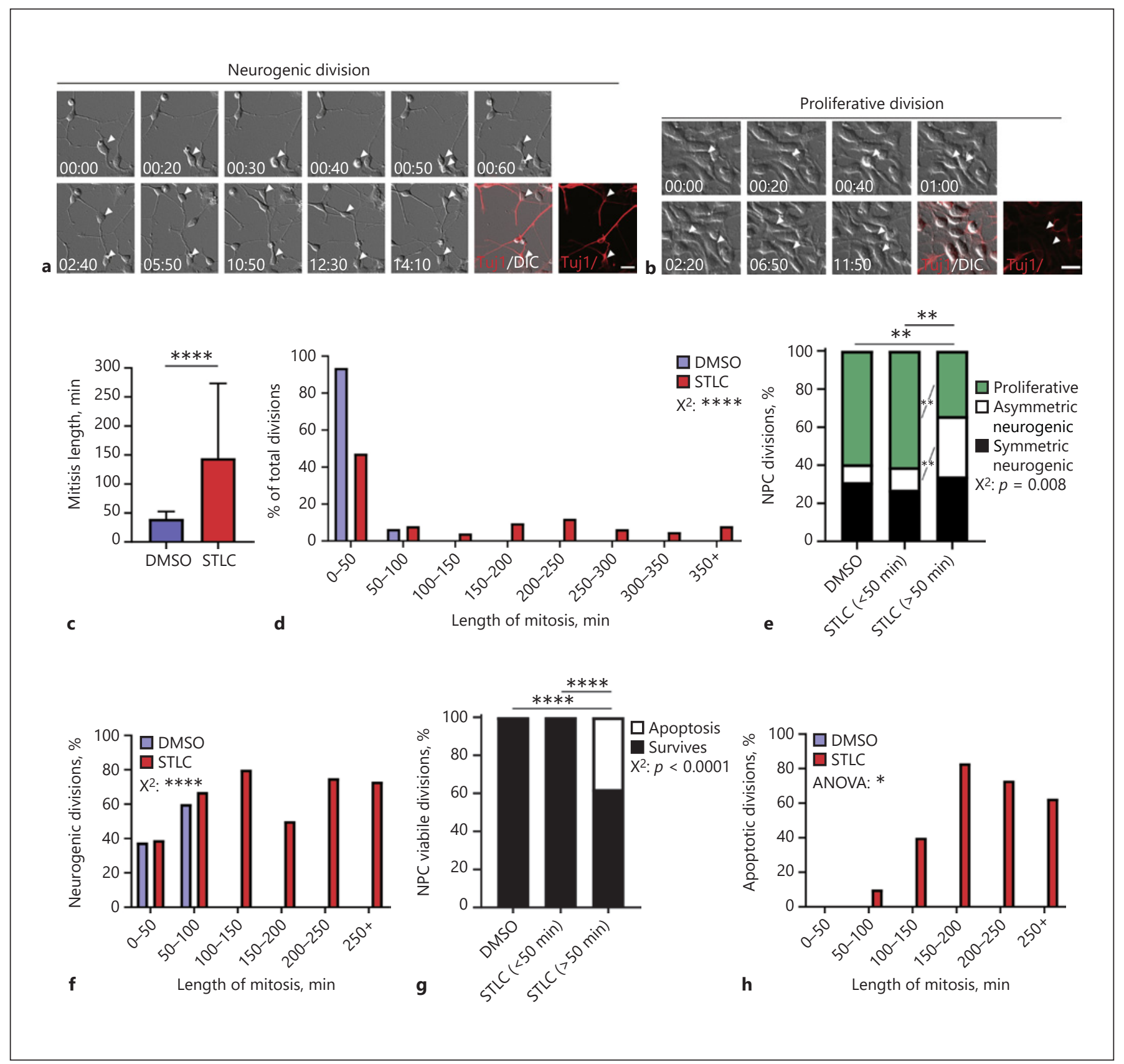

Fig. 6. Prolonged mitosis in human neural progenitors directly alters cell fate. a, b Panels of live imaging timeseries depicting neurogenic (a) and proliferative divisions (b) with immunostaining for Tuj1 (red) and DIC. Arrowheads indicate 1 cell which divides over time to become 2 cells. c Quantification of average mitosis duration for NPCs treated with either DMSO (blue) or STLC (red). Student's $t$ test. ${ }^{* * * *} p<0.0001$. d Histogram of NPC mitosis duration, using 50-min bins. $\chi^{2}$, **** $p<0.0001$. e Quantification of fraction of NPC divisions which are proliferative (Tuj1-; green), asymmetric neurogenic (1 Tuj1-, 1 Tuj1+, white), or symmetric neurogenic (both Tuj1+, black), under different conditions and graphed by mitosis duration. DMSO: $n=74$ daughter pairs, STLC $<50$ min: $n=58$ daughter pairs, STLC $>50$ min: $n=41$ cells. $\chi^{2}$ with
Bonferroni correction followed by post hoc analysis. DMSO vs. STLC delayed: ${ }^{* *} p=0.006$, STLC normal vs. delayed: ${ }^{* *} p=0.009$, post hoc proliferative fates: ${ }^{* *} p=0.001$, asymmetric neurogenic: ** $p=0.003$. $\mathbf{f}$ Histogram of neurogenic divisions ( $\%$ of total at each mitotic duration) in 50-min bins. $\chi^{2}$ : ${ }^{* * *} p<0.0001$. g Quantification of the fraction of NPC divisions generating viable (black) or apoptotic (white) progeny. DMSO: $n=74$ daughter pairs, STLC < 50 min: $n=58$ daughter pairs, STLC $>50$ min: $n=66$ cells. Statistics: $\chi^{2}$ and post hoc $\chi^{2}$ with Bonferroni correction. DMSO vs. delayed STLC: ${ }^{* * * *} p<0.0001$, STLC normal vs. delayed STLC: ${ }^{* * *} p<$ 0.0001 . $\mathbf{h}$ Histogram of proportion of apoptosis in progeny. ANOVA: ${ }^{*} p=0.02$. Error bar $=$ SD. Scale bar, $\mathbf{a}, \mathbf{b}, 20 \mu \mathrm{m}$. 
associated with prolonged mitosis. We generated Emx1Cre, $p 53^{l o x / l o x}$ mice and repeated experiments to prolong mitosis by acute injection of STLC or DMSO followed by FT $2 \mathrm{~h}$ later and harvesting brains at $+16 \mathrm{~h}$. In the absence of $p 53$ we noted no significant difference in Pax6 or NeuroD2+ cells between DMSO and STLC-treated p53 mutant brains (online suppl. Fig. S4C). These experiments suggest that within this in vivo paradigm, $\mathrm{p} 53$ signaling is activated and may influence daughter cells born from mitotically delayed progenitors.

Given the increase in p53 signaling and apoptosis following prolonged mitosis, we sought to understand upstream mechanisms triggering this cascade of events. One of the pathways associated with increased p53 signaling is DNA damage response, which was also upregulated in our transcriptome analysis. Our previous studies in vitro showed that DNA damage accumulates following mitotic delay, but it was unclear at which stage this occurred [11]. We thus assessed DNA damage in progenitors during prolonged prometaphase in vivo. For this we immunostained tissue sections with $\mathrm{\gamma H} 2 \mathrm{AX}$, a marker of doublestrand DNA breaks (Fig. 5e). FT+ and FT- RGCs were quantified 2 and $3 \mathrm{~h}$ after STLC or DMSO injection, in order to assess $\mathrm{\gamma H} 2 \mathrm{AX}$ levels in cells transiting mitosis. There was no difference in $\mathrm{\gamma H} 2 \mathrm{AX}$ signal in either $\mathrm{G} 2$ or prometaphase cells, indicating that DNA damage does not accumulate at the time of delay (Fig. 5f, g). In contrast, quantification of $\mathrm{\gamma H} 2 \mathrm{AX}+$ cells in metaphase and anaphase/telophase stages showed significant increases following STLC injection relative to the control (Fig. 5h, i). These experiments demonstrate that prometaphase delay in vivo results in increased DNA damage during metaphase and continuing into telophase.

Two pathways that are activated by DNA damage are ATM and ATR. We examined activation of the ATR pathway, using an antibody against phosphorylated $\mathrm{ATR}^{\mathrm{S} 345} ; 4 \mathrm{~h}$ after STLC injection we quantified higher pATR levels, suggesting that newborn progeny have activation of the ATR pathway (online suppl. Fig. S5A, B). We thus asked whether ATR activation explains altered cell fate following prolonged prometaphase. To inhibit ATR signaling, we used two well-characterized small molecules, AZ20 and VE821 [42, 43]. We first established conditions in which the drugs effectively inhibited ATR, as monitored by Western blot analysis. AZ20 or VE821 inhibition of ATR decreased pChk1 phosphorylation in mouse NIH3T3 cells (online suppl. Fig. S5C, D) and in primary cultures derived from E14.5 cortices (online suppl. Fig. S5E, F). To assess the impact of ATR signaling on cortical fates, we employed in vitro live imaging of pro-

Prolonged Progenitor Mitosis in Cortical Development genitors, an approach we have used previously $[11,44]$. Primary cells were live imaged with co-incubation of STLC or DMSO and select ATR inhibitors AZ20 and VE821. After $3 \mathrm{~h}$, STLC was washed out, and cells progressed through mitosis in the presence of ATR inhibitors for $3 \mathrm{~h}$, after which time cells were allowed to divide in normal medium. We examined fates of daughter cells at E13.5 from either delayed (STLC treated, $>50 \mathrm{~min}$ in mitosis) or not delayed (DMSO treated) progenitors cultured with AZ20 (1 $\mu \mathrm{M}$, control) or VE821 (160 nM) (online suppl. Fig. S5G, H). After $18 \mathrm{~h}$ we stained for cell fate markers Sox2, NeuroD2, and Tuj1, and apoptosis. By quantifying individual fate events, we noted no difference in either neurogenic divisions or viability regardless of whether STLC-delayed progenitors had ATR activity or not. This suggests that ATR activation does not explain apoptosis and cell fate changes following progenitor prolonged mitosis.

\section{Prolonged Mitosis Alters Fate of Human Neural Progenitors}

Our findings, together with previous studies, demonstrate that prolonged mitosis alters cell fate of mouse neuronal progeny in vivo, ex vivo, and in vitro. Finally, to further probe the relevance of prolonged mitosis for altered cell fate we asked whether NPCs show the same sensitivity to mitosis duration. We used human ESCs to generate cortical NPCs. These NPCs were plated onto polyornithine/laminin-coated 24-well plates in medium containing either DMSO (control) or STLC to induce prolonged mitosis. Progenitors were then imaged live, over a 24-h period, and DMSO or STLC was washed off after the first $4 \mathrm{~h}$ (Fig. 6a, b). We first quantified NPC mitosis duration, which significantly increased following STLC treatment (Fig. 6c). The vast majority of control NPCs proceeded to complete division within $50 \mathrm{~min}$ (Fig. 6d). Indeed, the average mitosis length for cells dividing in the STLC condition was $144 \mathrm{~min}$, and encompassed a range of mitosis lengths, from 40 to over 250 $\min$.

Fixed cells were then assessed for cell fate changes associated with mitotic delay. We quantified progeny expressing the neuronal marker Tuj1, and cell fates were retrospectively assigned to parental progenitors. Only in the delayed cohort did we observe increased neurogenic divisions and fewer proliferative divisions (Fig. 6e). In contrast, non-delayed human NPCs showed no difference in the neurogenic divisions compared to control (Fig. 6e). This reinforces that STLC acts upon cell fate by delaying NPCs in mitosis and does so with specificity. In

Dev Neurosci 2019;41:300-317 313 
the STLC conditions, however, there was an increased proportion of asymmetric neurogenic divisions compared to control or non-delayed divisions. Accordingly, symmetric proliferative divisions occurred at a significantly lower frequency across different mitotic durations (Fig. 6f). This suggests that mitotic delay of human NPCs resulted in significantly more neurogenic divisions at the expense of symmetric proliferative divisions.

We also assayed cell death by quantifying progeny cell morphology after $18 \mathrm{~h}$. Apoptosis occurred in 38\% of delayed cells and was absent in control divisions or in STLC conditions where divisions were shorter than $50 \mathrm{~min}$ (Fig. 6g). Again, we noted that apoptosis was evident across all mitotic durations with a significant increase that correlated with longer mitosis (Fig. 6h). These data demonstrate that human NPCs are subject to fate changes following prolonged mitosis in a similar manner to that seen in mice. Thus, cell biological and developmental programs responding to prolonged mitosis are conserved across species and relevant for human progenitors.

\section{Discussion}

Mutations in mitotic regulators result in human microcephaly, which has led to the long-standing model that mitotic defects underlie this disease. However, how perturbations to mitosis impact cortical development is unknown. In this study we demonstrate that acute prolonged mitosis of neural progenitors disrupts neurogenesis in vivo. We established and characterized a new experimental model for transiently and acutely lengthening progenitor mitosis and for monitoring cell fate. This experimental paradigm allowed us to gain new molecular insights linking prolonged mitosis to microcephaly-associated phenotypes. Further, we show that prolonged mitosis of human neural progenitors also alters cell fate, further supporting the value of investigating links between mitosis duration and fate of progeny. This experimental paradigm offers a valuable approach for interrogating mechanisms of microcephaly and related disorders. Further, our findings bolster the notion that mitotic delay of progenitors is a relevant mechanism to explain some cases of microcephaly.

Over 25 loci are implicated in the etiology of human microcephaly and a majority of these encode mitotic regulators [15]. This rich human genetics has continued to support the long-standing model that mitotic defects in progenitors underlie microcephaly [45-48]. Indeed, this notion has also been borne out experimentally. Across a variety of models including mice, organoids, and cultured cells, studies have shown that mitotic defects manifest in genetic and ZIKV etiology of microcephaly. However, very few studies have assessed how mitosis is perturbed. Most studies use only fixed analyses to show abundant mitotic cells, without normalizing this to progenitor cell cycle or number. In this regard, the use of live imaging, and detailed cell fate tracing in vivo, as we have done here, provides invaluable insights into the nature of mitotic defects. Along with prior studies [11], our data strongly argue that lengthened mitosis in mice in vivo and in human cells has detrimental impacts upon neurogenesis, and specifically abundant apoptosis, as well as imbalance of progenitors and neurons. Importantly, these same phenotypes are often evident in microcephaly models which co-present with mitotic delay.

In the future, implementation and variations on the experimental model we developed may be valuable to further understand the pathogenesis of microcephaly and to understand the role of mitotic delay in cell fate. There are several notable strengths of our model, as it allows one to acutely and reversibly delay RGCs in mitosis and then to trace cell fates in vivo. We also showed how this paradigm may be applied to different genetic backgrounds in order to interrogate genetic mechanisms by which prolonged mitosis impacts cell fate. Altogether, the neurogenesis defects we observed following acute mitotic delay in vivo were significant. It is important to note that the experiments in the current study used a single injection of STLC at E14.5, thus focusing the potential phenotypic impact to a single division. While technically challenging, it is exciting to consider how multiple injections across different stages could lead to a more potent and significant impact upon cortical lamination and brain size, thus further modeling a ZIKV or genetic microcephaly. Further, our studies indicate that higher dosages may have a more profound impact on mitosis duration. Likewise, it would be interesting to apply this acute delay model to earlier or later stages of development to evaluate roles for delayed progenitor mitosis in the neuroepithelial to RGC transition or astrocyte differentiation, respectively.

Our findings reinforce prior studies from our laboratory linking prolonged mitosis to cell fate in ex vivo and in vitro studies. The parallels we observe are remarkable given that the current experiments are all performed in vivo. There were some phenotypic differences; in comparison to slice experiments which led to 1.5 -fold more neurons and 1.25-fold fewer Tbr2 cells, the current experiments resulted in 1.2-fold more neurons, with no change in IPs [11]. Cell fate was comparable in DMSO- 
and STLC-treated $p 53$ mutants, whereas in comparison, in slices and primary cells, $p 53$ did not rescue cell fate following prolonged mitosis [11]. We posit that these phenotypic disparities may be attributable to differences in experimental paradigms. Here, we used a conditional $p 53$ model in which p53 is eliminated in RGCs at E9.5 with analysis at E14.5, whereas prior studies used a germline model and assayed cell fate one day earlier at E13.5. Neuronal fates in both studies were also monitored with different markers and different lineage tracers. Thus, experimental and phenotypic differences may explain the observed results. The $B A X^{-/-}$embryos retained variable cell death following prolonged mitosis, whereas p53 experiments suggest that apoptosis is a major cause of altered cell populations in this paradigm. However, quantification of equivalent apoptosis in progenitors and neurons suggests that the increase in neurons is not entirely explained by selective apoptosis.

\section{Mechanisms Linking Mitotic Delay to Microcephaly}

Our results consolidate a role for p53 signaling in microcephaly. Indeed, it is striking that across the literature, p53 is reproducibly identified as a unifying downstream pathway in microcephaly models [11, 41, 49-54]. Many genes mutated in primary microcephaly and microcephaly syndromes function in key aspects of mitosis including bipolar spindle formation and centrosome assembly (e.g., CEP152, CEP135, WDR62, CDK5RAP2, ASPM, SAS4/ CENPJ, CENPE, SAS6). Of these, in particular, loss of Sas4 results in a prolonged mitosis and p53 activation, and these mice have severe microcephaly [55]. Thus, from these data and this current work, we argue that prolonged mitosis is a plausible underlying cause of cell fate alterations in microcephaly. Indeed, this suggests that functional classification of microcephaly loci into those that lengthen mitosis, and particularly prometaphase, may be especially prone to the mechanisms described here.

A key question going forward is what are the signals that transduce the response to prolonged prometaphase, activating p53, altering cell fates, and potentiating apoptosis? We found that prolonged mitosis induces DNA damage in progenitors, followed by detectable pATR expression in newborn progeny, and p53 signaling within hours of cell birth, followed by cell death and altered neurogenic fates. Indeed, DNA damage, in certain contexts, can result in increased p53 activity and at times has been found to trigger cell cycle checkpoint arrest, or cell cycle exit. Here, we discover that increased DNA damage is upregulated in delayed progenitors, most notably at metaphase and anaphase. However, ATR inhibition did not

Prolonged Progenitor Mitosis in Cortical Development affect outcomes linked to delay. Thus, alternative pathways may explain why p53 upregulation occurs in newborn progeny.

A plausible path to discover pathways upstream of p53 is by interrogating molecular changes evident before p53 upregulation. Notably, our transcriptome analysis highlighted a number of candidates whose levels were significantly altered immediately following delay $(+2 \mathrm{~h})$. Amongst these are several transcripts associated with promoting neurogenesis, such as Neurogenin1, which would be of strong interest to interrogate further. Likewise, other omics-based approaches focused on changes at the protein level may also give valuable insights into possible translational and post-translational changes at play during mitotic delay.

Taken together, our study results further reinforce the notion that prolonged mitosis of progenitors has a deleterious impact on the developing brain. This has implications for developmental conditions like human microcephaly where prolonged mitosis is an important phenotypic occurrence.

\section{Acknowledgments}

We thank members of the Silver laboratory for insightful comments. We also thank Charlie Sheehan and Carly Newman for technical assistance with mouse husbandry. We thank the Duke genomics and bioinformatics cores for assistance.

\section{Statement of Ethics}

All mouse procedures were approved by the Duke Institutional Animal Care \& Use Committee (IACUC) and followed international guidelines.

\section{Disclosure Statement}

The authors have no conflicts of interest.

\section{Funding Sources}

This work was supported by the following: NIH R01NS083897, NIH R21NS098176, NIH R01NS110388, the Hartwell Foundation (D.L.S.); and NIH F31NS101785 (to A.M.-D.).

\section{Author Contributions}

A.M.-D., L.-J.P., and D.L.S. conceived of and designed the study. A.M.-D. and D.L.S. wrote the manuscript and all authors revised it. A.M.-D. carried out all experiments with assistance from A.C. 


\section{References}

1 Sousa AM, Meyer KA, Santpere G, Gulden FO, Sestan N. Evolution of the Human Nervous System Function, Structure, and Development. Cell. 2017 Jul;170(2):226-47.

2 Kowalczyk T, Pontious A, Englund C, Daza RA, Bedogni F, Hodge R, et al. Intermediate neuronal progenitors (basal progenitors) produce pyramidal-projection neurons for all layers of cerebral cortex. Cereb Cortex. 2009 Oct;19(10):2439-50.

3 Mihalas AB, Elsen GE, Bedogni F, Daza RA, Ramos-Laguna KA, Arnold SJ, et al. Intermediate Progenitor Cohorts Differentially Generate Cortical Layers and Require Tbr2 for Timely Acquisition of Neuronal Subtype Identity. Cell Rep. 2016 Jun;16(1):92-105.

4 Vasistha NA, García-Moreno F, Arora S, Cheung AF, Arnold SJ, Robertson EJ, et al. Cortical and Clonal Contribution of Tbr2 Expressing Progenitors in the Developing Mouse Brain. Cereb Cortex. 2015 Oct;25(10): 3290-302.

5 Takahashi T, Nowakowski RS, Caviness VS Jr. The cell cycle of the pseudostratified ventricular epithelium of the embryonic murine cerebral wall. J Neurosci. 1995 Sep;15(9):6046-57.

6 McConnell SK, Kaznowski CE. Cell cycle dependence of laminar determination in developing neocortex. Science. 1991 Oct;254(5029): 282-5.

7 Haydar TF, Ang E Jr, Rakic P. Mitotic spindle rotation and mode of cell division in the developing telencephalon. Proc Natl Acad Sci USA. 2003 Mar;100(5):2890-5.

8 Arai Y, Pulvers JN, Haffner C, Schilling B, Nüsslein I, Calegari F, et al. Neural stem and progenitor cells shorten S-phase on commitment to neuron production. Nat Commun. 2011 Jan;2(1):154.

9 Lange C, Huttner WB, Calegari F. Cdk4/cyclinD1 overexpression in neural stem cells shortens G1, delays neurogenesis, and promotes the generation and expansion of basal progenitors. Cell Stem Cell. 2009 Sep;5(3): 320-31.

10 Pilaz LJ, Patti D, Marcy G, Ollier E, Pfister S, Douglas RJ, et al. Forced G1-phase reduction alters mode of division, neuron number, and laminar phenotype in the cerebral cortex. Proc Natl Acad Sci USA. 2009 Dec;106(51): 21924-9.

11 Pilaz LJ, McMahon JJ, Miller EE, Lennox AL, Suzuki A, Salmon E, et al. Prolonged Mitosis of Neural Progenitors Alters Cell Fate in the Developing Brain. Neuron. 2016 Jan;89(1): 83-99.

12 Faheem M, Naseer MI, Rasool M, Chaudhary AG, Kumosani TA, Ilyas AM, et al. Molecular genetics of human primary microcephaly: an overview. BMC Med Genomics. 2015;8 Suppl $1: S 4$.

13 Gilmore EC, Walsh CA. Genetic causes of microcephaly and lessons for neuronal development. Wiley Interdiscip Rev Dev Biol. 2013 Jul;2(4):461-78.
14 Jayaraman D, Bae BI, Walsh CA. The Genetics of Primary Microcephaly. Annu Rev Genomics Hum Genet. 2018 Aug;19(1):177200.

15 Degrassi F, Damizia M, Lavia P. The Mitotic Apparatus and Kinetochores in Microcephaly and Neurodevelopmental Diseases. Cells. 2019 Dec;9(1):E49.

16 Zhang W, Yang SL, Yang M, Herrlinger S, Shao Q, Collar JL, et al. Modeling microcephaly with cerebral organoids reveals a WDR62CEP170-KIF2A pathway promoting cilium disassembly in neural progenitors. Nat Commun. 2019 Jun;10(1):2612.

17 Lancaster MA, Renner M, Martin CA, Wenzel D, Bicknell LS, Hurles ME, et al. Cerebral organoids model human brain development and microcephaly. Nature. 2013 Sep; 501(7467):373-9.

18 Marthiens V, Rujano MA, Pennetier C, Tessier S, Paul-Gilloteaux P, Basto R. Centrosome amplification causes microcephaly. Nat Cell Biol. 2013 Jul;15(7):731-40.

19 Silver DL, Watkins-Chow DE, Schreck KC, Pierfelice TJ, Larson DM, Burnetti AJ, et al. The exon junction complex component Magoh controls brain size by regulating neural stem cell division. Nat Neurosci. 2010 May; 13(5):551-8.

20 Asami M, Pilz GA, Ninkovic J, Godinho L, Schroeder T, Huttner WB, et al. The role of Pax6 in regulating the orientation and mode of cell division of progenitors in the mouse cerebral cortex. Development. 2011 Dec; 138(23):5067-78.

21 Gruber R, Zhou Z, Sukchev M, Joerss T, Frappart $\mathrm{PO}$, Wang $\mathrm{ZQ}$. MCPH1 regulates the neuroprogenitor division mode by coupling the centrosomal cycle with mitotic entry through the Chk1-Cdc25 pathway. Nat Cell Biol. 2011 Sep;13(11):1325-34.

22 Lizarraga SB, Margossian SP, Harris $\mathrm{MH}$, Campagna DR, Han A-P, Blevins S, et al. Cdk5rap2 regulates centrosome function and chromosome segregation in neuronal progenitors. Development. 2010 Jun;137(11): 1907-17.

23 McIntyre RE, Lakshminarasimhan Chavali P, Ismail O, Carragher DM, Sanchez-Andrade G, Forment JV, et al.; Sanger Mouse Genetics Project. Disruption of mouse Cenpj, a regulator of centriole biogenesis, phenocopies Seckel syndrome. PLoS Genet. 2012; 8(11):e1003022.

24 Yingling J, Youn YH, Darling D, Toyo-Oka K, Pramparo T, Hirotsune S, et al. Neuroepithelial stem cell proliferation requires LIS1 for precise spindle orientation and symmetric division. Cell. 2008 Feb;132(3):474-86.

25 Lazear HM, Diamond MS. Zika Virus: New Clinical Syndromes and Its Emergence in the Western Hemisphere. J Virol. 2016 Apr; 90(10):4864-75.

26 Li H, Saucedo-Cuevas L, Yuan L, Ross D, Johansen A, Sands D, et al. Zika Virus Pro- tease Cleavage of Host Protein Septin-2 Mediates Mitotic Defects in Neural Progenitors. Neuron. 2019 Mar 20;101(6):1089-98. e4.

27 Li R, Sun L, Fang A, Li P, Wu Q, Wang X. Recapitulating cortical development with organoid culture in vitro and modeling abnormal spindle-like (ASPM related primary) microcephaly disease. Protein Cell. 2017 Nov; 8(11):823-33.

28 Souza BS, Sampaio GL, Pereira CS, Campos GS, Sardi SI, Freitas LA, et al. Zika virus infection induces mitosis abnormalities and apoptotic cell death of human neural progenitor cells. Sci Rep. 2016 Dec;6(1):39775.

29 Wolf B, Diop F, Ferraris P, Wichit S, Busso C, Missé D, et al. Zika virus causes supernumerary foci with centriolar proteins and impaired spindle positioning. Open Biol. 2017 Jan;7(1): 160231.

30 Sheehan CJ, McMahon JJ, Serdar LD, Silver DL. Dosage-dependent requirements of Magoh for cortical interneuron generation and survival. Development. 2020 Jan; 147(1):dev182295

31 Shi Y, Kirwan P, Livesey FJ. Directed differentiation of human pluripotent stem cells to cerebral cortex neurons and neural networks. Nat Protoc. 2012 Oct;7(10):1836-46.

32 Skoufias DA, DeBonis S, Saoudi Y, Lebeau L, Crevel I, Cross R, et al. S-trityl-L-cysteine is a reversible, tight binding inhibitor of the human kinesin Eg5 that specifically blocks mitotic progression. J Biol Chem. 2006 Jun; 281(26):17559-69.

33 Telley L, Govindan S, Prados J, Stevant I, Nef $\mathrm{S}$, Dermitzakis E, et al. Sequential transcriptional waves direct the differentiation of newborn neurons in the mouse neocortex. Science. 2016 Mar;351(6280):1443-6.

34 Govindan S, Oberst P, Jabaudon D. In vivo pulse labeling of isochronic cohorts of cells in the central nervous system using FlashTag. Nat Protoc. 2018 Oct;13(10):2297-311.

35 Namba T, Huttner WB. Neural progenitor cells and their role in the development and evolutionary expansion of the neocortex. Wiley Interdiscip Rev Dev Biol. 2017 Jan;6(1): $1-16$.

36 Olson JM, Asakura A, Snider L, Hawkes R, Strand A, Stoeck J, et al. NeuroD2 is necessary for development and survival of central nervous system neurons. Dev Biol. 2001 Jun; 234(1):174-87.

37 Vitali I, Fievre S, Telley L, Oberst P, Bariselli S, Frangeul L, et al. Progenitor Hyperpolarization Regulates the Sequential Generation of Neuronal Subtypes in the Developing Neocortex. Cell. 2018;174(5):1264-76. e15.

38 Greig LC, Woodworth MB, Galazo MJ, Padmanabhan H, Macklis JD. Molecular logic of neocortical projection neuron specification, development and diversity. Nat Rev Neurosci. 2013 Nov;14(11):755-69. 
39 Southwell DG, Paredes MF, Galvao RP, Jones DL, Froemke RC, Sebe JY, et al. Intrinsically determined cell death of developing cortical interneurons. Nature. 2012 Nov;491(7422):109-13.

40 Quintens R, Verreet T, Janssen A, Neefs M, Leysen L, Michaux A, et al. Identification of novel radiation-induced $\mathrm{p} 53$-dependent transcripts extensively regulated during mouse brain development. Biol Open. 2015 Feb;4(3): 331-44.

41 Mao H, McMahon JJ, Tsai YH, Wang Z, Silver DL. Haploinsufficiency for Core Exon Junction Complex Components Disrupts Embryonic Neurogenesis and Causes p53-Mediated Microcephaly. PLoS Genet. 2016 Sep;12(9): e1006282-27.

42 Foote KM, Blades K, Cronin A, Fillery S, Guichard SS, Hassall L, et al. Discovery of 4-\{4-[(3R)-3-Methylmorpholin-4-yl]-6-[1(methylsulfonyl)cyclopropyl]pyrimidin2-yl\}-1H-indole (AZ20): a potent and selective inhibitor of ATR protein kinase with monotherapy in vivo antitumor activity. J Med Chem. 2013 Mar;56(5):2125-38.

43 Prevo R, Fokas E, Reaper PM, Charlton PA, Pollard JR, McKenna WG, et al. The novel ATR inhibitor VE-821 increases sensitivity of pancreatic cancer cells to radiation and che- motherapy. Cancer Biol Ther. 2012 Sep; 13(11):1072-81.

44 Pilaz L-J, Silver DL. Live imaging of mitosis in the developing mouse embryonic cortex. J Vis Exp. 2014(88):e51298-e.

45 Bond J, Roberts E, Mochida GH, Hampshire DJ, Scott S, Askham JM, et al. ASPM is a major determinant of cerebral cortical size. Nat Genet. 2002 Oct;32(2):316-20.

46 Jackson AP, Eastwood H, Bell SM, Adu J, Toomes C, Carr IM, et al. Identification of microcephalin, a protein implicated in determining the size of the human brain. Am J Hum Genet. 2002 Jul;71(1):136-42.

47 Kumar A, Markandaya M, Girimaji SC. Primary microcephaly: microcephalin and ASPM determine the size of the human brain. J Biosci. 2002 Dec;27(7):629-32.

48 Mochida GH. Genetics and biology of microcephaly and lissencephaly. Semin Pediatr Neurol. 2009 Sep;16(3):120-6.

49 Little JN, Dwyer ND. p53 deletion rescues lethal microcephaly in a mouse model with neural stem cell abscission defects. Hum $\mathrm{Mol}$ Genet. 2019 Feb;28(3):434-47.

50 Shi L, Qalieh A, Lam MM, Keil JM, Kwan KY. Robust elimination of genome-damaged cells safeguards against brain somatic aneuploidy following Knl1 deletion. Nat Commun. 2019 Jun;10(1):2588

51 Ghouzzi V, Bianchi FT, Molineris I, Mounce BC, Berto GE, Rak M, et al. ZIKA virus elicits P53 activation and genotoxic stress in human neural progenitors similar to mutations involved in severe forms of genetic microcephaly and p53. Cell Death Dis. 2017 Jan 19;8(1):e2567.

52 Breuss M, Fritz T, Gstrein T, Chan K, Ushakova $\mathrm{L}, \mathrm{Yu}$ N, et al. Mutations in the murine homologue of TUBB5 cause microcephaly by perturbing cell cycle progression and inducing p53-associated apoptosis. Development. 2016 Apr;143(7):1126-33.

53 Marjanović M, Sánchez-Huertas C, Terré B, Gómez R, Scheel JF, Pacheco S, et al. CEP63 deficiency promotes p53-dependent microcephaly and reveals a role for the centrosome in meiotic recombination. Nat Commun. 2015 Jul 9;6:7676.

54 Insolera R, Bazzi H, Shao W, Anderson KV, Shi SH. Cortical neurogenesis in the absence of centrioles. Nat Neurosci. 2014 Nov; 17(11): 1528-35.

55 Bazzi H, Anderson KV. Acentriolar mitosis activates a p53-dependent apoptosis pathway in the mouse embryo. Proc Natl Acad Sci USA. 2014 Apr;111(15):E1491-500. 